\title{
Simulation of light-weight membrane structures by wrinkling model
}

\author{
Riccardo Rossi ${ }^{1}$, Massimiliano Lazzari ${ }^{1}$, Renato Vitaliani $^{1, *, \dagger}$ and Eugenio Oñate ${ }^{2}$ \\ ${ }^{1}$ Department of Structural and Transportation Engineering, University of Padua, 35131 Padua, Italy \\ ${ }^{2}$ International Center for Numerical Methods in Engineering (CIMNE), Universidad Politécnica de Cataluña, \\ Campus Norte UPC, 08034 Barcelona, Spain
}

\begin{abstract}
SUMMARY
The computational challenge in dealing with membrane systems is closely connected to the lack of bending stiffness that constitutes the main feature of this category of structures. This manifests numerically in badly conditioned or singular systems requiring the use of stabilized solution procedures, in our case of a 'pseudo-dynamic' approach. The absence of the flexural stiffness makes the membrane very prone to local instabilities which manifest physically in the formation of little 'waves' in 'compressed' areas. Current work presents an efficient, sub-iteration free 'explicit', penalty material based, wrinkling simulation procedure suitable for the solution of 'static' problems. The procedure is stabilized by taking full advantage of the pseudo-dynamic solution strategy, which allows to retain the elemental quadratic convergence properties inside the single solution step. Results are validated by comparison with published results and by setting up 'numerical experiments' based on the solution of test cases using dense meshes. Copyright (c) 2005 John Wiley \& Sons, Ltd.
\end{abstract}

KEY WORDS: geometrical non-linear behaviour; membrane structures; wrinkling

\section{INTRODUCTION}

When the ratio between the thickness and the other dimensions of a structural shell systems gets very low, the flexural contribution to the response to the external loads loses importance, making appealing the use of a mathematical model which neglects the bending contribution, namely a purely membrane model. The immediate consequence of such an idealization is that the motion of the structure outside its own plane is not restrained making possible both 'global' rigid body motions and 'local' instabilities (e.g. References [1-3]).

This behaviour is physical, particularly it can be readily verified that real membranes show local instability phenomena, made evident by the formation of little waves in zones in which

\footnotetext{
*Correspondence to: R. Vitaliani, Department of Structural and Transportation Engineering, University of Padua, 35131 Padua, Italy.

${ }^{\dagger}$ E-mail: rvit@ caronte.dic.unipd.it
}

Copyright (c) 2005 John Wiley \& Sons, Ltd.

Received 9 June 2003

Revised 13 October 2004

Accepted 13 October 2004 
compression tends to appear. The phenomena manifests as a consequence of the formation of compressive stresses as in this conditions any initial imperfection immediately leads to an out of plane displacement which reduces the resistance of the membrane to the compression (making the compression vanish). The shape of those 'waves' or 'wrinkles' is governed by the state of stress and by the local bending stiffness of the system, high bending resistance resulting in few large 'waves', low resistance in numerous (at the limit infinite) little wrinkles.

Generally speaking the kinematic model for a FE membrane formulation does not allow the description of wrinkles of size smaller then the element's dimension. This results in a non-physical compressive resistance shown by 'coarse' FE models.

Correct description of the wrinkles is possible by increasing the mesh density up to a level at which the single wave is subdivided between a number of elements. This approach is possible and needed when accurate prediction of the wrinkle's size and distribution is needed; different authors References [4-6] addressed the problem by using rotation free thin shells models. Numerical prediction of the wrinkle's size and distribution together with experimental validation can be found in References [7-10] with reference to space structures, for which the formation of the wrinkles becomes a crucial aspect of the structural behaviour. This works address the simulation of the so-called 'Kapton's membrane' by the use of ABAQUS S4R5 fournodes shell elements, describing in detail the effect of initial imperfections and the loading and unloading behaviour of the different systems. Such appronches allow an excellent description of the system's deform to the expected size for large systems.

In many cases on the other hand a 'deterministic' predictio needed or the system's the winkles which ma
unknowns (construction
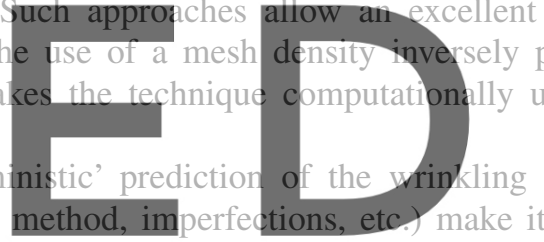

In those cases it is of interest to obtain a sort of 'averaged membrane response' in the form

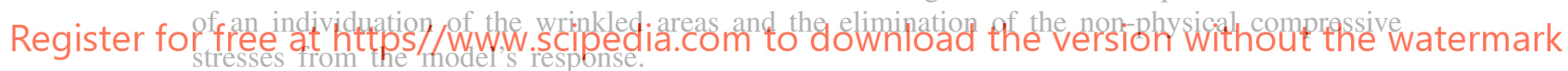

It is worth noting that this approach is not necessarily less precise than the previous one: indeed no information on the wrinkling size is provided, but the giobal stress field is properiy described. As well it is important to highlight how in civil engineering structures, the position of wrinkles is never known because of its strong dependence on the initial imperfections; in this case the only reliable result is the identification of the wrinkled areas.

Over the years this problem was addressed using basically two different strategies: a first one $[11,27]$ based on the modification of the deformation gradient to take in account the formation of the wrinkles, a second one taking in account the formation of the wrinkles by modifying the constitutive law.

It is commonly recognized that the introduction of the wrinkling leads often to numerical instabilities ('... tension field behaviour may exhibit erratic behaviour during the solution process and possibly prevent convergence..' [12]). In the authors experience the inclusion of the wrinkling phenomena as an additional source of non-linearity leads to a slower convergence of the Newton-Raphson algorithm or eventually to a 'high' number of subiterations. An increase in the number of iterations was reported in the literature [11] even for the case in which the consistent tangent operator was used.

In current work we address an efficient 'static' solution procedure for membrane systems including the wrinkling correction. Our procedure is based on a penalty material model which 
is similar to the one proposed in Reference [12] the main innovation being connected to the solution strategy used.

It is well known that straight-forward 'static' solution of membrane systems is not possible because of impending local rigid body motions which make the stiffness matrix badly conditioned or singular. The use of a 'pseudo-static' solution procedure based on an equivalent zero-mass damped dynamical system allows smooth convergence to the static solution. This procedure allows the determination of the final 'static' configuration by performing a number of 'dynamic steps' which should be intended as 'artificial states' without real physical meaning.

Our technique is based on an 'explicit' correction of the material which is kept constant inside each solution step. No guarantee is given that the compressions are correctly removed at the end of each solution step, however when the dynamic procedure converged to a static configuration no further change for the strain field manifests which ensures convergence of the wrinkling procedure.

It should be noted that keeping the material constant inside each solution step ensures that the convergence properties of the membrane finite element are kept, follows immediately that the only additional cost is connected to the modification of the constitutive law.

Stability of the procedure is greatly increased by the presence of the dynamic terms, a stabilization for the material modification phase is however proposed which allows the oscillations of the stress field to be damped out in a shorter time.
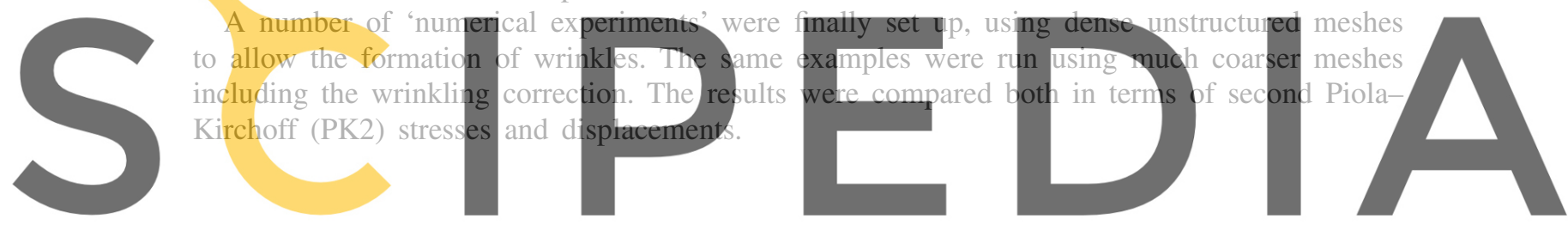

2. ELEMENT TECHNOLOGY

Register for free at htt.ps//www.scipedia.com to download the version without the watermark The problem discussed here is connected with the description of the motion of thin structures in the space. In the analysis large displacements have to be taken in account because the problem is geometrically non-linear. The non-linear finite element procedure is developed according to the total Lagrangian formulation using appropriate stress and strain measures as the GreenLagrange strain and the PK2 stress (e.g. References [13-15]). Forms of material non-linearity can be introduced at a later stage.

Focus is here given on the formulation of the elements to be used in the analysis. The crucial point is that the stress field is contained in the plane (or axis) of the element, but the element itself can rotate in the $3 \mathrm{D}$ space. As a result, the contribution to the equilibrium is connected with its actual orientation.

From a practical point of view this means that the constitutive law can be formulated in terms of in-plane (or axial) strains and of in-plane stresses. This is greatly simplified by the introduction of a co-rotational system of co-ordinates.

Although it is possible to formulate higher order elements, it is well known that in presence of severe distortions low-order elements are much more reliable (e.g. Reference [16]). Therefore stress is given to the formulation of an efficient triangular 3D membrane element.

The reference paper for the formulation used is Reference [17], while details on the derivation of the various terms involved can be found in References $[18,19]$. Next lines give a briefly outline of the formulation used, together with the basic steps needed for the implementation. 
As mentioned in the opening, a convenient co-rotational system of axis is introduced. In this system of co-ordinates the deformation of the element becomes a two-dimensional plane stress problem [14]. The main advantage of the present formulation in comparison with a classical co-rotational approach is that no explicit change of base is needed, allowing a greater efficiency to be achieved.

Given the vectors $\mathbf{x}^{1}, \mathbf{x}^{2}, \mathbf{x}^{3}\left(\mathbf{x}^{I}=\left\{x_{1}^{I} x_{2}^{I} x_{3}^{I}\right\}^{\mathrm{T}}\right.$ is the co-ordinate of the node ' $\left.I^{\prime}\right)$ describing the current position of the nodes in the global co-ordinate system, Figure 1, it is useful to define the vectors:

$$
\mathbf{x}^{21}=\mathbf{x}^{2}-\mathbf{x}^{1}, \quad \mathbf{x}^{31}=\mathbf{x}^{3}-\mathbf{x}^{1}, \quad \mathbf{x}^{32}=\mathbf{x}^{3}-\mathbf{x}^{2}
$$

Taking advantage of this notation a local co-ordinate system can be defined by:

$$
\mathbf{v}_{1}=\frac{\mathbf{x}^{21}}{\left\|\mathrm{x}^{21}\right\|}, \quad \mathbf{v}_{3}=\left(\mathbf{x}^{21}\right) \times\left(\mathrm{x}^{31}\right), \quad \mathrm{n}=\frac{\mathrm{v}_{3}}{\left\|\mathrm{v}_{3}\right\|}, \quad \mathbf{v}_{3}=2 \mathrm{Am}, \quad \mathrm{v}_{2}=(\mathrm{n}) \times\left(\mathrm{v}_{1}\right)
$$

Vectors $\mathbf{v}_{1}, \mathbf{v}_{2}, \mathbf{m}$ form an orthonormal base with $\mathbf{v}_{1}$ and $\mathbf{v}_{2}$ describing the plane of the element. $A$ is the current area of the element and the relations between $\mathbf{v}_{3}$ and $\mathbf{n}$ is a consequence of the properties of the vector produce $(\times)[20]$.

The representation of the position vectors (for a given node ' $I$ ') in the local co-ordinate $\left(\mathbf{v}_{1}, \mathbf{v}_{2}, \mathbf{n}\right)$ system is
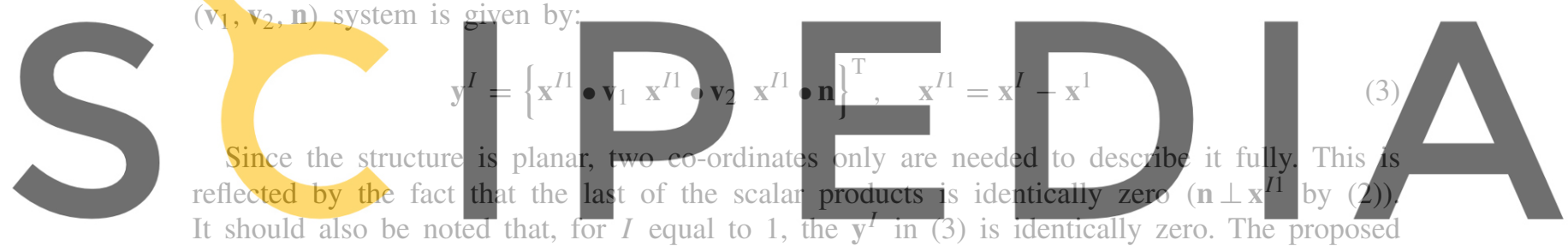
co-ordinate system is defined during all of the deformation process.

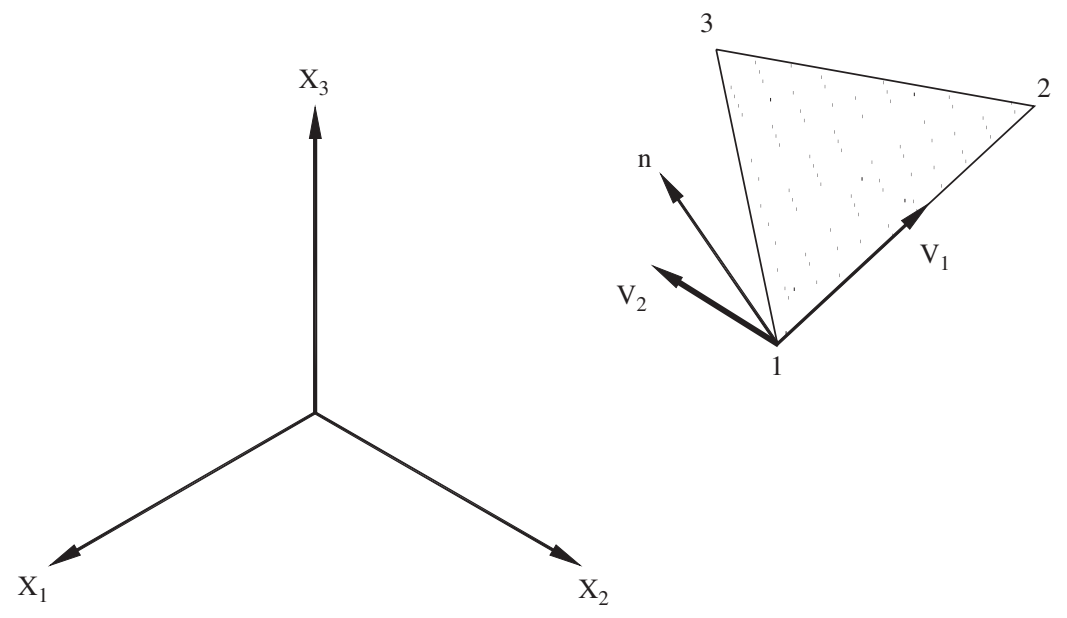

Figure 1. Co-ordinate systems used with membrane element. 


\subsection{Kinematic model}

In order to develop the formulation of membrane element, two assumptions are made: that displacement field inside each element is constrained in the plane of the element itself and that approximation for the displacement field is linear.

The use of area co-ordinates $\xi_{1}, \xi_{2}$, and $\xi_{3}$ allows to express conveniently the position of any point inside the membrane $\left(\mathbf{y}^{1}=\mathbf{0} \rightarrow \xi_{1} \mathbf{y}^{1}=\mathbf{0}, \mathbf{Y}^{1}=\mathbf{0} \rightarrow \xi_{1} \mathbf{Y}^{1}=\mathbf{0}\right.$, Figure 1) as:

$$
\mathbf{y}=\xi_{2} \mathbf{y}^{2}+\xi_{3} \mathbf{y}^{3}, \quad \mathbf{Y}=\xi_{2} \mathbf{Y}^{2}+\xi_{3} \mathbf{Y}^{3}
$$

In order to go further in the analysis of the deformation process, it is useful to take in account the deformation gradient $\mathbf{F}$; this can be opportunely done by the introduction of the two tensors $\mathbf{j}(\mathbf{j}=\partial \mathbf{y} / \partial \xi)$ and $\mathbf{J}(\mathbf{J}=\partial \mathbf{Y} / \partial \boldsymbol{\xi})$ :

$$
\mathbf{F}=\frac{\partial \mathbf{y}}{\partial \mathbf{Y}}=\frac{\partial \mathbf{y}}{\partial \xi} \frac{\partial \xi}{\partial \mathbf{Y}}=\mathbf{j J}^{-1}
$$

Note that $\mathbf{F}$ is a $2 \times 2$ tensor even if work is developed in a three-dimensional environment (the three-dimensional problem is here reduced to a two-dimensional one by working in a suitable co-ordinate system). This observation is crucial for the success of the formulation.

Trace of the rigid body motion of the element in the 3D space (translation and rotation) is

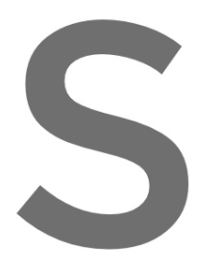
kept in the definition rigid body motions.

\section{Register for}
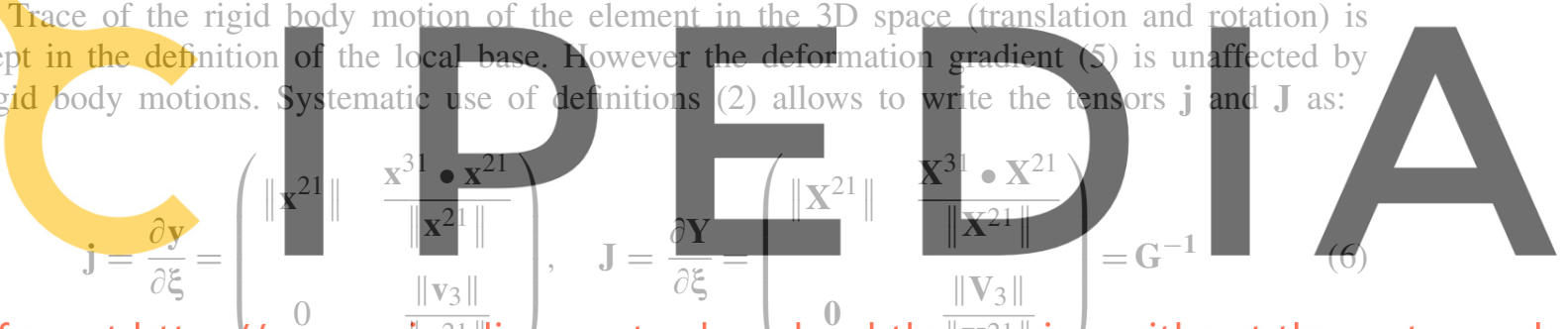

It should be noted that $\mathrm{G}=\mathrm{J}^{-1}$ is independent from the deformation and can be calculated once and stored. In writing (6) it was taken in account that:

$$
\mathbf{y}^{2}=\left(\begin{array}{c}
\mathbf{x}^{21} \bullet \mathbf{v}_{1} \\
\mathbf{x}^{21} \bullet \mathbf{v}_{2}
\end{array}\right)=\left(\begin{array}{c}
\left\|\mathbf{x}^{21}\right\| \\
0
\end{array}\right), \quad \mathbf{y}^{3}=\left(\begin{array}{c}
\mathbf{x}^{31} \bullet \mathbf{v}_{1} \\
\mathbf{x}^{31} \bullet \mathbf{v}_{2}
\end{array}\right)=\left(\begin{array}{c}
\frac{\mathbf{x}^{31} \bullet \mathbf{x}^{21}}{\left\|\mathbf{x}^{21}\right\|} \\
\frac{\left\|\mathbf{v}_{3}\right\|}{\left\|\mathbf{x}^{21}\right\|}
\end{array}\right)
$$

in which it was used the relation $\mathbf{v}^{3}=\left\|\mathbf{x}^{21}\right\|\left(\mathbf{x}^{31} \bullet \mathbf{v}^{2}\right) \mathbf{v}^{1} \times \mathbf{v}^{2} \rightarrow \mathbf{x}^{31} \bullet \mathbf{v}^{2}=\left\|\mathbf{v}^{3}\right\| /\left\|\mathbf{x}^{21}\right\|$. Introducing the tensor $\mathbf{g}$ as:

$$
\mathbf{g}=\mathbf{j}^{\mathrm{T}} \mathbf{j}=\left(\begin{array}{cc}
\left\|\mathbf{x}^{21}\right\|^{2} & \mathbf{x}^{31} \bullet \mathbf{x}^{21} \\
\mathbf{x}^{31} \bullet \mathbf{x}^{21} & \left(\frac{\mathbf{x}^{31} \bullet \mathbf{x}^{21}}{\left\|\mathbf{x}^{21}\right\|}\right)^{2}+\frac{\mathbf{v}_{3} \bullet \mathbf{v}_{3}}{\left\|\mathbf{x}^{21}\right\|^{2}}
\end{array}\right)=\left(\begin{array}{cc}
\mathbf{x}^{21} \bullet \mathbf{x}^{21} & \mathbf{x}^{31} \bullet \mathbf{x}^{21} \\
\mathbf{x}^{31} \bullet \mathbf{x}^{21} & \mathbf{x}^{31} \bullet \mathbf{x}^{31}
\end{array}\right)
$$


the Green-Lagrange strain tensor can be properly expressed as:

$$
[\mathbf{E}]=\frac{1}{2}\left([\mathbf{F}]^{\mathrm{T}}[\mathbf{F}]-[\mathbf{I}]\right)=\frac{1}{2}\left([\mathbf{G}]^{\mathrm{T}}[\mathbf{g}][\mathbf{G}]-[\mathbf{I}]\right)
$$

and its variation in the form:

$$
[\delta \mathbf{E}]=\frac{1}{2}[\mathbf{G}]^{\mathrm{T}}[\delta \mathbf{g}][\mathbf{G}]
$$

Switching to Voigt Notation, (10) assumes then the form:

$$
\{\delta \mathbf{E}\}=\frac{1}{2}[\mathbf{Q}]\{\delta \mathbf{g}\}=[\mathbf{Q}][\mathbf{b}]\{\delta \mathbf{x}\}=[\mathbf{B}]\{\delta \mathbf{x}\}
$$

where matrix [Q] is obtained between using the indexes map $\begin{gathered}i I, j J \rightarrow a b \\ G_{i I} G_{j J} \rightarrow Q_{a b}\end{gathered}$ which links $\mathbf{G}$ to $\mathbf{Q}$, $\{\delta \mathbf{g}\}$ is derived from and (8) and $[\mathbf{B}]$ as

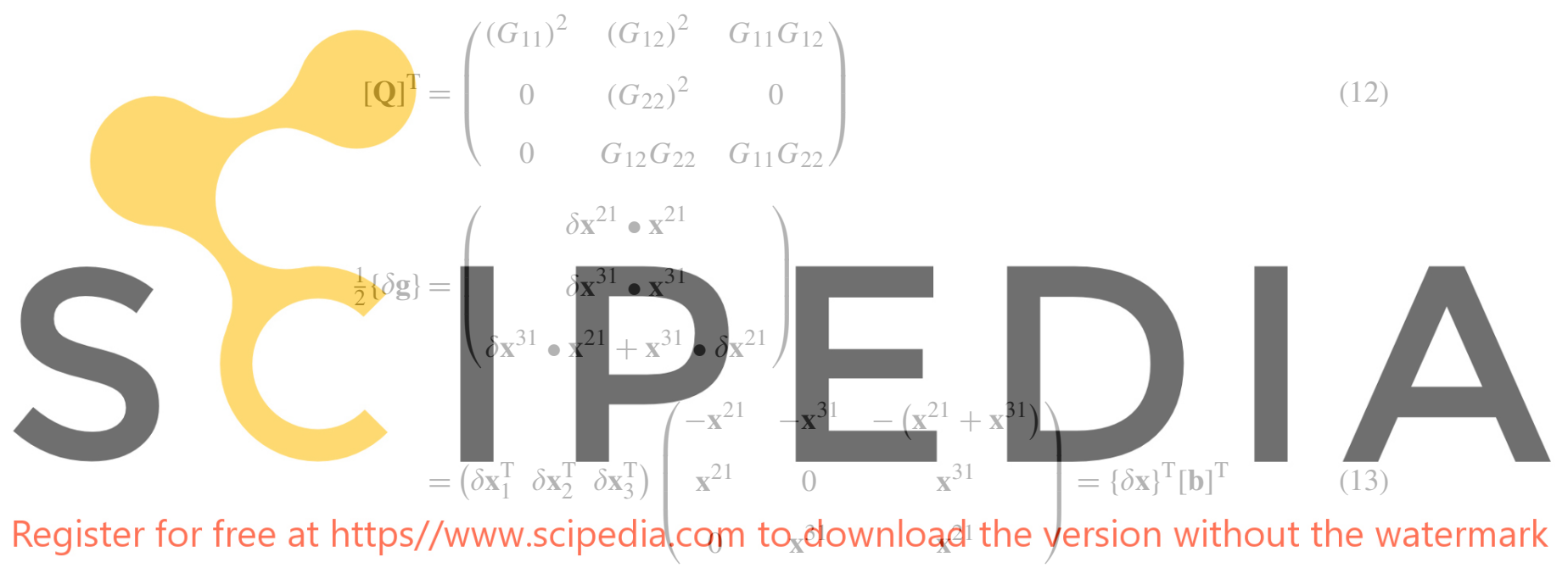

$$
[\mathrm{B}]=[\mathrm{Q}][\mathrm{b}]
$$

\subsection{Principle of virtual works}

The balance between the internal and external work $\left(W_{\mathrm{int}}, W_{\mathrm{b}_{\mathrm{ext}}}, W_{\mathrm{t}}\right.$ are the internal, external and prescribed traction works) takes the form:

$$
W_{\text {int }}=\int_{\Omega} \frac{h_{0}}{2}\{\delta \mathbf{E}\}^{\mathrm{T}}\{\mathbf{S}\} \mathrm{d} \Omega=\int_{\omega} \delta \mathbf{u} \bullet \mathbf{b} \mathrm{d} \omega+\int_{\gamma} \delta \mathbf{u} \bullet \mathbf{t} \mathrm{d} \gamma=W_{\mathrm{b}_{\mathrm{ext}}}+W_{\mathrm{t}}
$$

where $\{\mathbf{S}\}=\left(\begin{array}{lll}S_{11} & S_{22} & S_{12}\end{array}\right)^{\mathrm{T}}$ is the PK2 stress, $\Omega$ is the area in the reference configuration and $\omega$ and $\gamma$ are area and boundary in the current configuration, $h$ is the thickness of membrane, $\mathbf{b}$ and $\mathbf{t}$ are body and prescribed traction vector. Applying (10), the internal work (15) appears as:

$$
\left\{\mathbf{f}_{\text {int }}\right\}=\int_{\Omega} \frac{h_{0}}{2}\{\delta \mathbf{E}\}^{\mathrm{T}}\{\mathbf{S}\} \mathrm{d} \Omega=h_{0} A_{0}\{\delta \mathbf{x}\}^{\mathrm{T}}[\mathbf{B}]^{\mathrm{T}}\{\mathbf{S}\}
$$

The element under description is non-linear which makes necessary the evaluation of the tangent terms in order to complete the formulation. This is done within the framework given 
Table I. Step of the calculations.

\begin{tabular}{lcccc}
\hline Step $1 \rightarrow$ & $2 \rightarrow$ & $3 \rightarrow$ & $4 \rightarrow$ & $5 \rightarrow$ \\
$\left\{\mathbf{x}^{I J}\right\}, i=1,2,3 ; j=1,2(1)$ & $\left\{\mathbf{v}_{3}\right\}(2)$ & {$[\mathbf{Q}]^{t}(12)$} & {$[\mathbf{b}]^{\prime}(13)$} & {$[\mathbf{B}](14)$} \\
Step 6 $\rightarrow$ & $7 \rightarrow$ & $8 \rightarrow$ & $9 \rightarrow$ & $10 \rightarrow$ \\
{$[\mathbf{j}](6)$} & {$[\mathbf{g}](8)$} & $\left\{\mathbf{f}_{\text {int }}\right\}(16)$ & {$[\mathbf{K m}](22)$} & {$[\mathbf{K g}](20)$} \\
\hline
\end{tabular}

by the Newton-Raphson procedure $\left\{\mathbf{x}^{k+1}\right\}=\left\{\mathbf{x}^{k}\right\}+\{\mathrm{d} \mathbf{x}\}$. Starting from the definition of a residual force work, $\{\boldsymbol{\psi}\}=\left\{\mathbf{f}_{\text {ext }}\right\}-\left\{\mathbf{f}_{\text {int }}\right\}$, the tangents can be evaluated by:

$$
\left(\frac{\partial\left\{\mathbf{f}_{\text {int }}\right\}}{\partial\{\mathbf{x}\}}-\frac{\partial\left\{\mathbf{f}_{\text {ext }}\right\}}{\partial\{\mathbf{x}\}}\right)_{\{\mathbf{x}\}=\left\{\mathbf{x}^{k}\right\}} \mathrm{d}\{\mathbf{x}\}=\left\{\psi\left(\left\{\mathbf{x}^{k}\right\}\right)\right\}
$$

The differentiation of the internal force term takes the form:

$$
\mathrm{d}\left(\mathbf{f}_{\text {int }}\right)=\mathrm{d}(\int_{\Omega} \frac{h_{0}}{2}\left\{\delta \mathbb{E}^{\mathrm{T}}\{\mathbf{S}\} \mathrm{d} \Omega\right)=\underbrace{\frac{A_{0} h_{0}}{2} \mathrm{~d}\left(\{\delta \mathbf{E}\}^{\mathrm{T}}\right)\{\mathbf{S}\}}_{\text {Geometric stiffness }}+\underbrace{\frac{A_{0} h_{0}}{2}\{\delta \mathbf{E}\}^{\mathrm{T}} \mathrm{d}(\{\mathbf{S}\})}_{\text {Material stiffness }}
$$
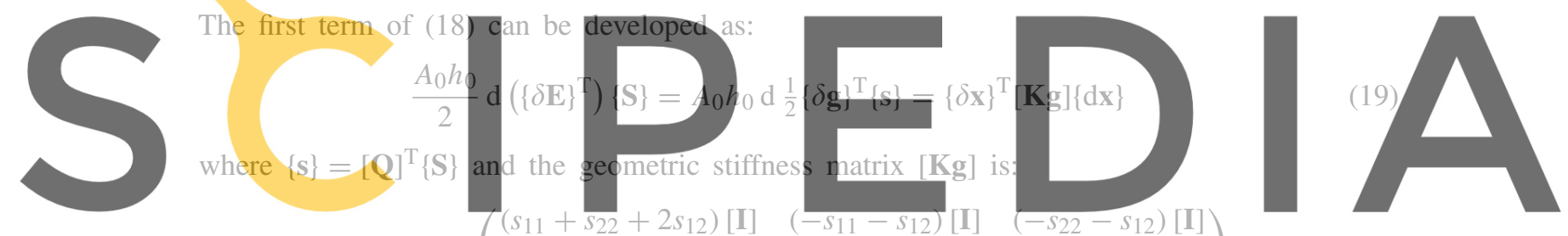

Register for free at

The second term in Equation (18) can be developed applying (10) as:

$$
\frac{A_{0} h_{0}}{2}\{\delta \mathbf{E}\}^{\mathrm{T}} \mathrm{d}(\{\mathbf{S}\})=\frac{A_{0} h_{0}}{2}\{\mathrm{~d} \mathbf{x}\}^{\mathrm{T}}[\mathbf{B}]^{\mathrm{T}}\left[\mathbf{D}_{T}\right][\mathbf{B}]\{\delta \mathbf{x}\}=\{\mathrm{d} \mathbf{x}\}^{\mathrm{T}}\left[\mathbf{K}_{\text {mat }}\right]\{\delta \mathbf{x}\}
$$

where $\{\mathrm{d} \mathbf{S}\}=\left[\mathbf{D}_{T}\right][\mathbf{B}]\{\delta \mathbf{x}\}$ and the material tangent stiffness matrix $\left[\mathbf{K}_{\text {mat }}\right]$ is:

$$
\left[\mathbf{K}_{\text {mat }}\right]=h_{0} A_{0}[\mathbf{B}]^{\mathrm{T}}\left[\mathbf{D}_{T}\right][\mathbf{B}]
$$

For linear elastic material $\left[\mathbf{D}_{T}\right]$ simply coincides with the normal $[\mathbf{D}]$ and is valid with reference to total strains and stresses and not only in incremental form. It is worth stressing how membrane structures are classically characterized by large displacements with associated small strains. Linear material models are then generally suitable for the descriptions of the material behaviour.

The following two points summarize the steps to be performed for the evaluation of the terms needed:

1. to calculate and to store $G$ by Equation (6) one time;

2. to perform steps in Table I at each iteration. 


\section{DYNAMIC PROCEDURE: QUASI-STATIC SOLUTION}

The use of Newton-Raphson approach allows to formulate the non-linear analysis through the repeated solution of a linear system. The solution relies therefore on the hypothesis that the stiffness matrix is non-singular, which is connected with the impossibility of rigid body motions. As the resistance of our structures to 'out-of-plane' loads relies purely on the ability to reach a deformed geometric configuration, rigid body motions are clearly not prevented and a straightforward application of the Newton-Raphson procedure is not possible.

On the other hand, the presence of rigid body motions does not represent a problem when a dynamic analysis is performed. It could be therefore appealing to obtain the static solution as the limit of a dynamic (damped) solution of the same problem (e.g. References [21,22]). This approach is 'physical' and can lead to very good results. Unfortunately when the damping is low, many steps are needed for the oscillations to be damped out, which could lead to very long (computationally expensive) analyses. The ideal choice is to damp critically the structure in order to reach the steady state without oscillations.

An attractive alternative to the full dynamic simulation is the use of a pseudo-dynamic analysis, obtained by setting the mass matrix to zero and using an arbitrary damping matrix. In these conditions the system is automatically subjected to super-critical damping and no transient has to be expected. A possible choice for the damping matrix is $\mathbf{C}_{\text {damp }}=\alpha \mathbf{M} ; \alpha=1$ was found to be a good choice in many cases, higher values shoutd be used for the case of very thin membranes.

Simulations perfor tioning for the stiffne use and discuss other

It is therefore of interest to
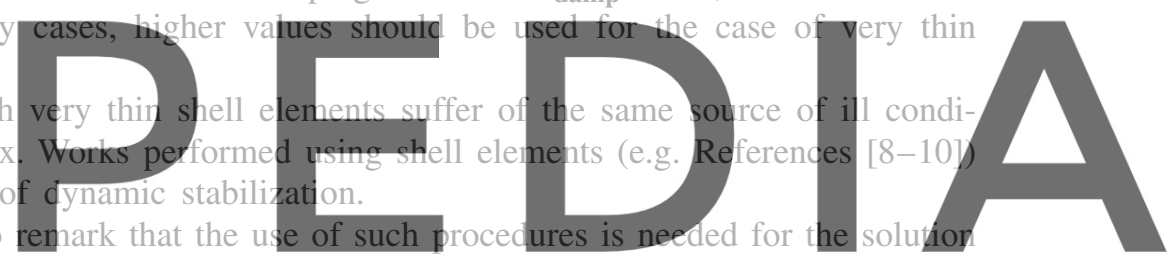

of the membrane problem and should not be intended as an additional unnecessary cost.

Register for free atynamics, is taken in accgunt (17) hecomes:

$$
\psi_{\text {dyn }}\left({ }^{t} \mathbf{x}\right)=\mathbf{f}_{\text {ext }}\left({ }^{t} \mathbf{x}\right)-\mathbf{f}_{\text {int }}\left({ }^{t} \mathbf{x}\right)-\mathbf{f}_{\text {kin }}\left({ }^{t} \mathbf{x}\right)-\mathbf{f}_{\text {damp }}\left({ }^{t} \mathbf{x}\right)
$$

where ${ }^{t} \mathbf{x}=\mathbf{x}(t)$. Using this notation the Newton-Raphson procedure assumes the form:

$$
\left(\left.\frac{\partial \boldsymbol{\psi}}{\partial \mathbf{x}}\right|_{\mathbf{x}=t^{\prime} \mathbf{x}^{k}}+\left.\frac{\partial \mathbf{f}_{\mathbf{k i n}}}{\partial \mathbf{x}}\right|_{\mathbf{x}=t^{t} \mathbf{x}^{k}}+\left.\frac{\partial \mathbf{f}_{\mathbf{d a m p}}}{\partial \mathbf{x}}\right|_{\mathbf{x}=t_{\mathbf{x}^{k}}}\right) \mathrm{d} \mathbf{x}=\psi\left({ }^{t} \mathbf{x}^{k}\right)-\mathbf{f}_{\mathbf{k i n}}\left({ }^{t} \mathbf{x}\right)-\mathbf{f}_{\mathbf{d a m p}}\left({ }^{t} \mathbf{x}\right)
$$

Classically it is assumed that mass and damping matrixes are constant during the deformation (linear damping term). The actual evaluation of these terms is then connected to the time integration scheme used here. Newmark scheme is based on the approximations (e.g. References [13, 14]):

$$
\begin{aligned}
{ }^{t} \ddot{\mathbf{x}}^{i+1} & =\frac{1}{\beta \Delta t^{2}}\left({ }^{t} \dot{\mathbf{x}}^{i+1}-{ }^{t-\Delta t} \mathbf{x}\right)-\frac{1}{\beta \Delta t}{ }^{t-\Delta t} \dot{\mathbf{x}}-\left(\frac{1}{2 \beta}-1\right){ }^{t-\Delta t} \ddot{\mathbf{x}} \\
{ }^{t} \dot{\mathbf{x}}^{i+1} & =\frac{\gamma}{\beta \Delta t}\left({ }^{t} \mathbf{x}^{i+1}-{ }^{t-\Delta t} \mathbf{x}\right)-\left(\frac{\gamma}{\beta}-1\right){ }^{t-\Delta t} \dot{\mathbf{x}}-\frac{\Delta t}{2}\left(\frac{\gamma}{\beta}-2\right){ }^{t-\Delta t} \ddot{\mathbf{x}}
\end{aligned}
$$


where $\beta$ and $\gamma$ are two parameters which describe the form of the approximation. The elemental tangent matrix $\mathbf{K}_{\tan }$ and the RHS take then the form:

$$
\begin{aligned}
\mathbf{K}_{\text {tan }_{\text {el }}} & =\mathbf{K}_{\text {static }}+\frac{\gamma}{\beta \Delta t} \mathbf{C}_{\text {damp }}+\frac{1}{\beta \Delta t^{2}} \mathbf{M} \\
\mathbf{b}_{\tan _{\text {el }}}{ }^{t}{ }^{k} & =\mathbf{b}_{\text {static }}{ }^{t} \mathbf{x}^{k}-\mathbf{C}_{\text {damp }}{ }^{t} \dot{\mathbf{x}}^{k}-\mathbf{M}^{t} \ddot{\mathbf{x}}^{k}
\end{aligned}
$$

Classical values for the parameters are $\beta=1 / 4$ and $\gamma=1 / 2$ (with $\gamma>1 / 2$ a numerical damping is added to the solution of the dynamic system).

\section{ELEMENT ENRICHMENT}

Over the years many different proposals to perform the element enrichment were developed (e.g. References [23-25]). Mainly two different approaches survived: one based on manipulations of the gradient of deformations (kinematics manipulation), the second connected with a redefinition of the constitutive model (material manipulation).

The former, proposed by Roddeman et al. [26,27] and used for example in Reference [11], is based on the definition of an effective deformation gradient obtained by superimposing to the normal displacement allows to describe in in presence of compr on the modification always smooth. In an non-linearity in a problem that is already highly non-linear.

The second approach is based on a modification of the stress-strain relationship, so that the

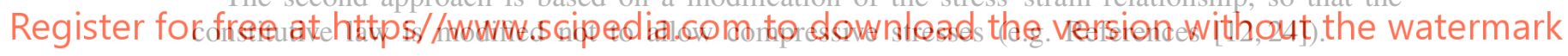

The main advantage of these second techniques is to make the implementation completely independent from the element used, feature that makes them very attractive for the practical implementation. In addition, they can be easily modified to deal with anisotropy materials. The current work describes in full detail the implementation of an enriched material model, providing a discussion of the difficulties and of the strategies developed to overcome them.

The modified material model used is derived from the model proposed in Reference [12] from which differs in some details concerning the determination of the state. The main difference is that in the literature the material is treated as non-linear modifying iteratively the elasticity tensor during the Newton-Raphson iterations while in this case no iterative correction on the material state is performed. In other words the material is kept constant over the whole solution step which ensures that the convergence properties of the element are kept (compressive stress may still exist at the end of the step).

\subsection{Material model}

The following pages describe a new material model based on the modification of a standard linear material. The material is softened in the direction of the principal stresses if compression appears. 
This is obtained by the two following steps:

- Evaluation of the 'state' from the original configuration of the membrane.

- Modification of the material.

The state of the membrane can be defined taut state, Figure 2(a), when both the principal stresses are positive, wrinkled state, Figure 2(b), when the smaller principal strain is negative but the smaller principal stress is zero, and slack state, Figure 2(c), when both principal stresses are negative.

Figure 3 shows the three state in two airbags. It is possible to observe zones with taut and wrinkled state in Figure 3(a) and zones with slack state in Figure 3(b).

In order to proceed farther, a distinction between the original and modified material is necessary. In particular the following symbols will be adopted:

- [D]: constitutive tensor for the original material, without any penalization;

- $\left[\mathbf{D}_{\mathrm{mod}}\right]$ : constitutive tensor for the modified material or penalized material.

Thus there will be a fictitious stress state $\left[\sigma^{*}\right]$ (linked with $[\mathbf{D}]$ ) and an effective one $[\sigma]$ (linked with $\left[\mathbf{D}_{\bmod }\right]$ ) acting on the structure.

In the development of the current material, model any path dependence is completely neglected, making unnecessary the incremental formulations of stresses and strains. This is
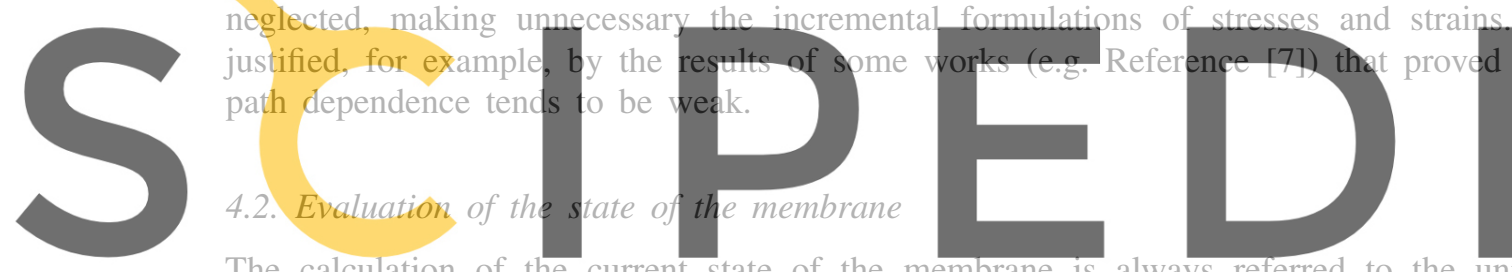

The calculation of the current state of the membrane is always referred to the unmodified material $[\mathbb{D}]$, so the history dependence is ignored. The procedure is explained with the following Register forsfęe at https//www.scipedia.com to download the version without the watermark

- Analysis is performed using $\left[\mathbb{D}_{\bmod }\right]$, the total strain $[\varepsilon]$ is evaluated and passed to the material model.

- Fictitious stresses $\left[\boldsymbol{\sigma}^{*}\right]$ (representing the stresses that would appear if the original membrane would be subjected to the same deformation) are calculated using the unmodified material [D]:

$$
\left[\boldsymbol{\sigma}^{*}\right]=[\mathbf{D}][\varepsilon]
$$

Principal stresses are calculated together with the orientation of the principal system, Figure 4(a):

$$
\begin{aligned}
& c_{1}=\left(\sigma_{11}^{*}+\sigma_{22}^{*}\right) / 2, \quad c_{2}=\sigma_{11}^{*}-\sigma_{22}^{*}, \quad c_{3}=\sqrt{\left(c_{2} / 2\right)^{2}+\left(\sigma_{12}^{*}\right)^{2}} \\
& \sigma_{1}^{*}=c_{1}+c_{3}, \quad \sigma_{2}^{*}=c_{1}-c_{3}, \quad 2 \alpha^{*}=\tan ^{-1}\left(2 \sigma_{12}^{*} / c_{2}\right)
\end{aligned}
$$

corresponding strains are calculated projecting the strains in the system of the principal axis evaluated by stress matrix (they are not necessarily the principal ones for anisotropy materials), 


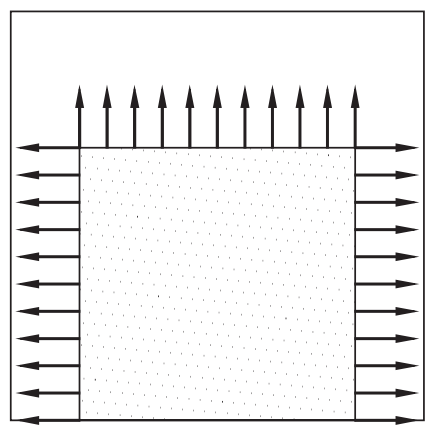

(a)
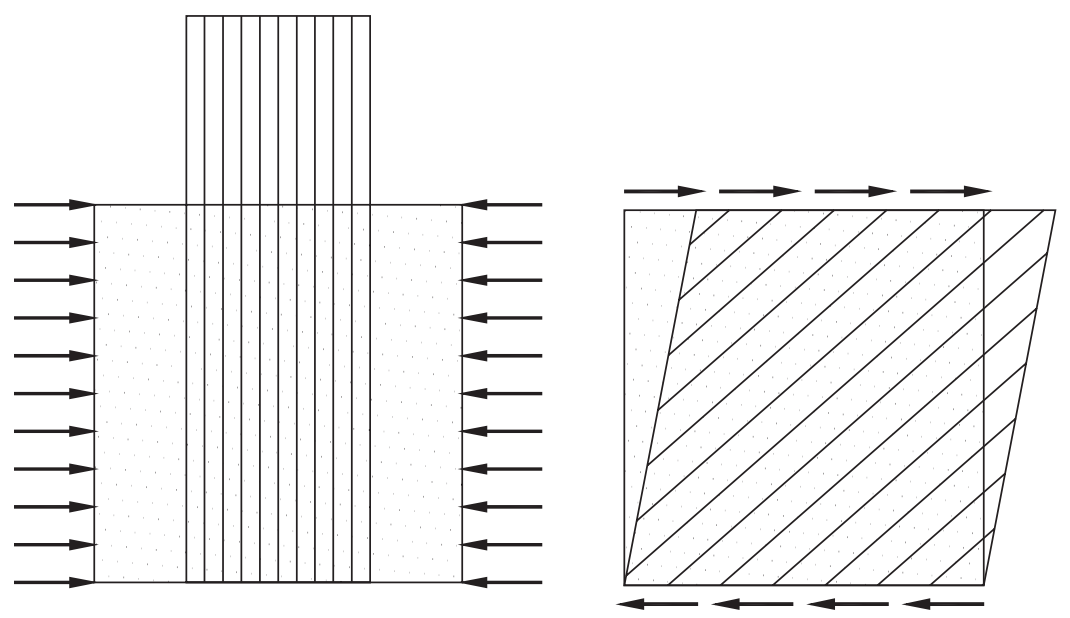

(b)
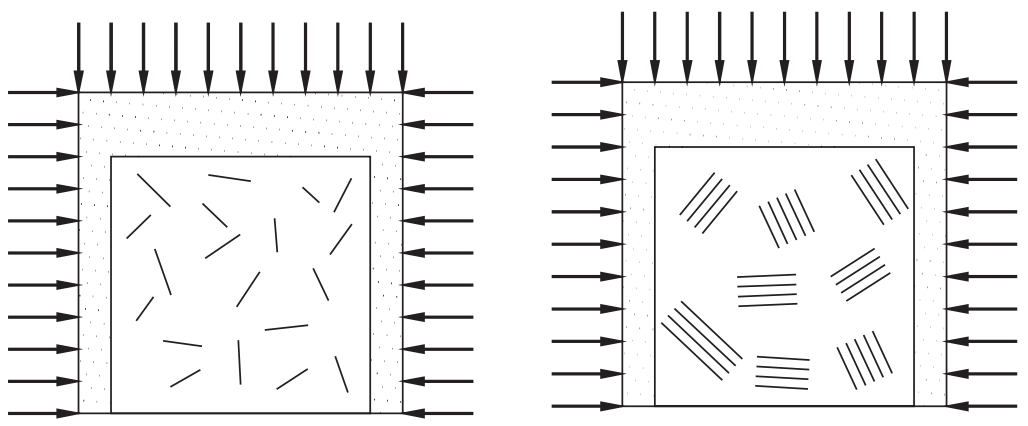

(c)

Figure 2. Membrane state: (a) 'taut state'; (b) 'wrinkled state'; and (c) irregular wrinkles or 'slack' state. 


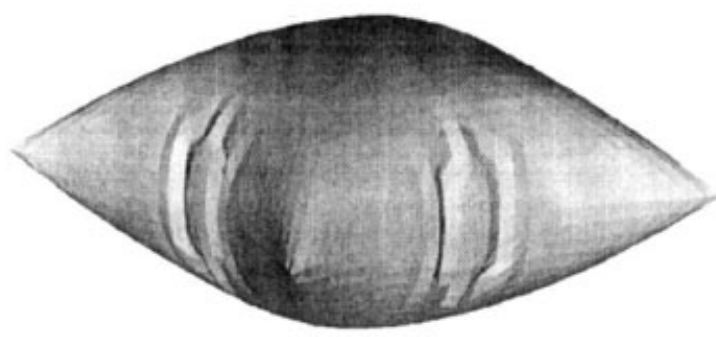

(a)

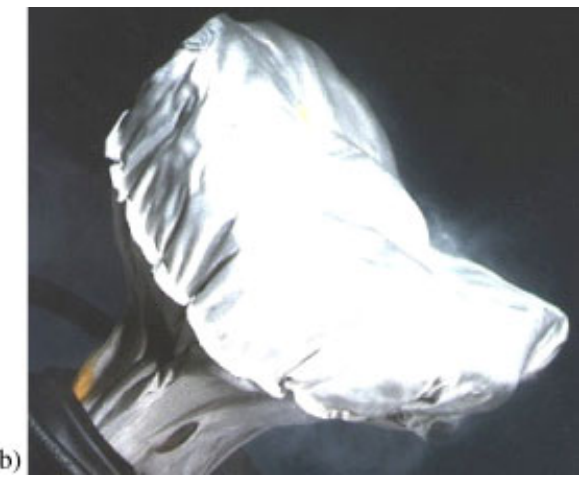

Figure 3. Airbag membrane: (a) inflated; and (b) initial inflated.

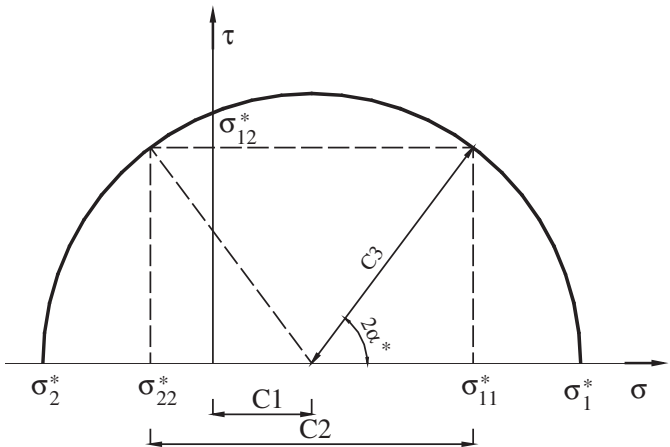

(a)

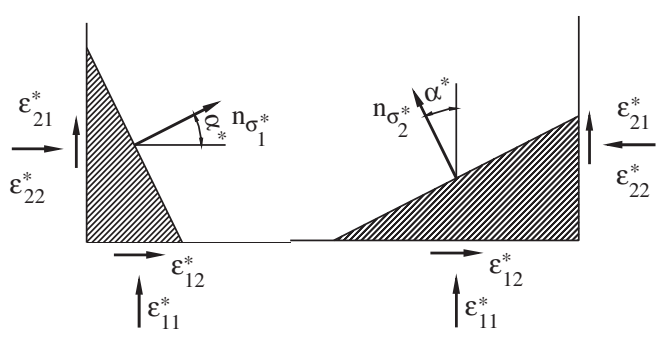

(b)

Figure 4. (a) Mohr circle for stress fictions tensor; and (b) strain component.

see Figure 4(b):

$$
\begin{aligned}
& {\left[\mathbf{n}_{\boldsymbol{\sigma}_{1}^{*}}\right]=\left[\begin{array}{c}
\cos \left(\alpha^{*}\right) \\
\sin \left(\alpha^{*}\right)
\end{array}\right], \quad\left[\mathbf{n}_{\boldsymbol{\sigma}_{2}^{*}}\right]=\left[\begin{array}{c}
-\sin \left(\alpha^{*}\right) \\
\cos \left(\alpha^{*}\right)
\end{array}\right]} \\
& \varepsilon_{\sigma_{1}^{*}}=\left[\mathbf{n}_{\boldsymbol{\sigma}_{1}^{*}}\right]^{\mathrm{T}}[\boldsymbol{\varepsilon}]\left[\mathbf{n}_{\boldsymbol{\sigma}_{1}^{*}}\right]=\left(\varepsilon_{11} \cos \alpha^{*}+\frac{\gamma_{12}}{2} \sin \alpha^{*}\right) \cos \alpha^{*}+\left(\frac{\gamma_{12}}{2} \cos \alpha^{*}+\varepsilon_{22} \sin \alpha^{*}\right) \sin \alpha^{*} \\
& \varepsilon_{\sigma_{2}^{*}}=\left[\mathbf{n}_{\boldsymbol{\sigma}_{2}^{*}}\right]^{\mathrm{T}}[\boldsymbol{\varepsilon}]\left[\mathbf{n}_{\boldsymbol{\sigma}_{2}^{*}}\right]=\left(\varepsilon_{11} \sin \alpha^{*}-\frac{\gamma_{12}}{2} \cos \alpha^{*}\right) \sin \alpha^{*}+\left(\varepsilon_{22} \cos \alpha^{*}-\frac{\gamma_{12}}{2} \sin \alpha^{*}\right) \cos \alpha^{*}
\end{aligned}
$$

Equation (32) is the normal component of the strain to the surface with $\left[\mathbf{n}_{\boldsymbol{\sigma}_{\mathbf{1}}^{*}}\right]$ and $\left[\mathbf{n}_{\mathbf{\sigma}_{\mathbf{2}}^{*}}\right]$ as a normal vector, see Figure 4(b).

- Evaluation of the membrane state is carried out.

Different kind of criteria, based on stress and strain, can be used to assess the state of the membrane [12] as taut state (completely in tension), wrinkled state (only one direction in tension) and slack state (completely in compression): 
- Stress criterion, Figure 2(a), is based upon the principal fictitious stress (30):

$$
\begin{array}{ll}
\text { case } \sigma_{2}^{*}>0: & \text { 'taut state' } \\
\text { case } \sigma_{2}^{*} \leqslant 0 \text { and } \sigma_{1}^{*}>0: & \text { 'wrinkled state' } \\
\text { otherwise: } & \text { 'slack state' }
\end{array}
$$

The application of this criterion is straightforward, although in some cases the condition of 'slackness' is misjudged. This can be seen for example considering in the space of the principal strains (Figure 2(a)) a point $E_{1} E_{2}$ located between the axis $E_{2}$ and the line with slope $-1 / v$. A biaxial compression is associated to this strain, corresponding to a slack state with the criteria above, while as the principal strain is positive the real state is wrinkled.

- Strain criterion, Figure 2(b), is based upon the normal strain defined by the principal stress direction (32), only:

$$
\begin{array}{ll}
\text { case } \varepsilon_{\sigma_{2}}^{*}>0: & \text { 'taut state' } \\
\text { case } \varepsilon_{\sigma_{2}^{*}} \leqslant 0 \text { and } \varepsilon_{\sigma_{1}^{*}}>0: & \text { 'wrinkled state' } \\
\text { otherwise: } & \text { 'slack state' }
\end{array}
$$

Also this simple criterion shows some difficult points, because-while describing accurately the state of the membrane-it misjudges the idea of 'tautness'. It is quite possible to have positive minimum stress and negative strain due to the effect of Poisson's ratio [12].

- Mixed criterion, Figure 2(c), is defined by stress and strain:

$$
\begin{array}{ll}
\text { case } \sigma_{2}^{*}>0: & \text { 'taut state' completely in tension } \\
\text { case } \sigma_{2}^{*}<0 \text { and } \varepsilon_{\sigma_{1}^{*}}>0: & \text { 'wrinkled state' only one direction in tension } \\
\text { otherwise: } & \text { 'slack state' completely in compression }
\end{array}
$$

The last criterion, developed by Roddeman et al. (e.g. References [26, 27]), allows to overcome the difficulties illustrated above. This criterion, based on both stresses and strains, provides the most accurate description of a real membrane state and has been therefore chosen for the implementation of the current algorithm.

The main difference between the proposed technique and previous works on the subject is that the check of the stress state and the identification of the state is carried out in the membrane with original material $[\mathbf{D}]$ and not with the modified material $\left[\mathbf{D}_{\text {mod }}\right]$. In other words the solution is computed using the modified material but in the assessment of the strains the unmodified material is considered. We remark that the deformations are assumed to be small which allows to use effectively the PK2 stresses instead of the Cauchy ones in writing the correction procedure.

\subsection{Penalization of the material}

The standard procedure for the modification of the constitutive matrix, $\left[\mathbf{D}_{\text {mod }}\right]$ is described by using the penalty factor $P$. Assuming that the state of the membrane is calculated with the 
stresses $\left(\left[\sigma^{*}\right]\right)$ and with the orientation of the principal system $\left(\alpha^{*}\right)$ the following procedure can be defined:

- taut state:

$$
\left[\mathbf{D}_{\text {mod }}\right]=[\mathbf{D}]
$$

- wrinkled state: calculation of the constitutive matrix $\left[\mathbf{D}_{\text {rot }}\right]$ in the system of principal stresses $\left[\boldsymbol{\sigma}^{*}\right]$ :

$$
\begin{aligned}
c & =\cos \left(\alpha^{*}\right), \quad s=\sin \left(\alpha^{*}\right), \quad c_{2}=c^{2}, \quad s_{2}=s^{2} \\
s c & =s * c, \quad\left[\mathbf{R}\left(\alpha^{*}\right)\right]=\left[\begin{array}{ccc}
c_{2} & s_{2} & -2 s c \\
s_{2} & c_{2} & 2 s c \\
s c & -s c & c_{2}-s_{2}
\end{array}\right] \\
{\left[\mathbf{D}_{\mathrm{rot}}\right] } & =\left[\mathbf{R}\left(-\alpha^{*}\right)\right][\mathbf{D}]\left[\mathbf{R}\left(-\alpha^{*}\right)\right]^{\mathrm{T}}
\end{aligned}
$$

penalization of $\left[\mathbf{D}_{\text {rot }}\right]$ by the penalty factor $P$ :

$$
\left[\mathbf{D}_{\mathrm{rot}, \mathrm{mod}}\right]=\left[\begin{array}{ccc}
C_{\mathrm{rot}, 11} & P \cdot C_{\mathrm{rot}, 12} & C_{\mathrm{rot}, 13} \\
P \cdot C_{\mathrm{rot}, 21} & P \cdot C_{\mathrm{rot}, 22} & P \cdot C_{\mathrm{rot}, 23} \\
C_{\mathrm{rot}, 31} & P \cdot C_{\mathrm{rot}, 32} & C_{\mathrm{rot}, 33}
\end{array}\right]
$$

back to the standard system of co-ordinates with calculation of the constitutive matrix $\left[\mathbf{D}_{\text {mod }}\right]$ :

$$
\left[\mathbf{D}_{\text {mod }}\right]=\left[\mathbf{R}\left(\alpha^{*}\right)\right]\left[\mathbf{D}_{\text {rot, } \bmod }\right]\left[\mathbf{R}\left(\alpha^{*}\right)\right]^{\mathrm{T}}
$$

- slack state:

$$
\left[\mathbf{D}_{\text {mod }}\right]=P[\mathbf{D}]
$$

In the reference article [12] the penalization parameter $P$ is a constant. Unfortunately, without any further modification, a pretty long time is needed for the stress oscillations to damp out.

A source of instability is connected with the redistribution of stresses caused by the softening of certain directions during the analysis. This often causes a cyclic change in the state of some elements whose contribution to stiffness is basically switched on (taut state) and off (slack state).

Stability (and particular speed in stabilizing the stress field) is improved by the following modifications:

- Defining the penalty parameter $P$ as a function of the maximum $\left(\sigma_{\max }\right)$ and effective $\left(\sigma_{2}\right)$ compressive stress (allowing a small compression in the structure) into (45) and (47).

$$
P_{\sigma}=\frac{\sigma_{\max }}{\sigma_{2}} \rightarrow \begin{cases}P_{\sigma}<P & \rightarrow P=P \\ P_{\sigma}>1 \text { or } P_{\sigma}<0 \rightarrow P=1.0\end{cases}
$$



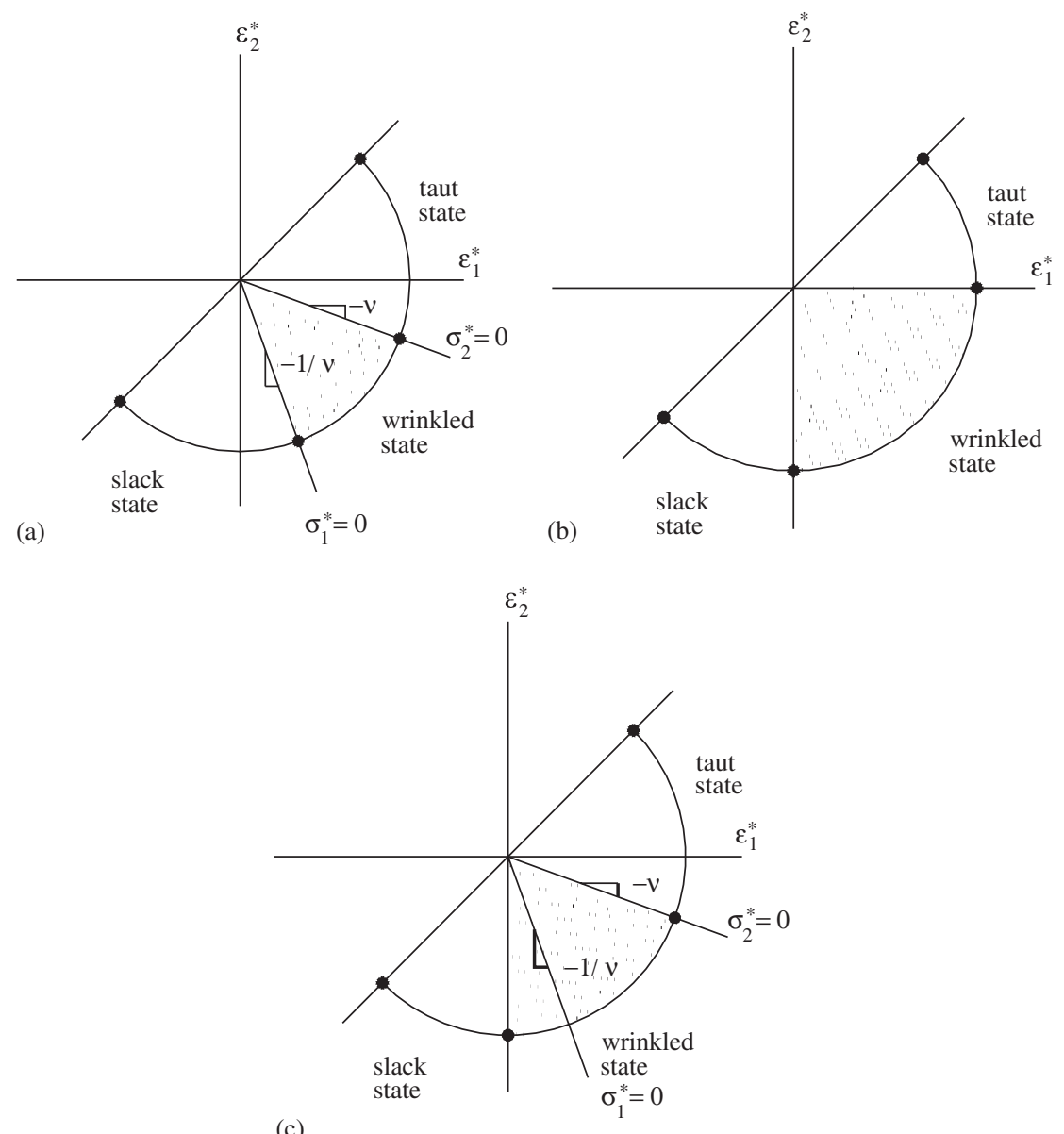

(c)

Figure 5. Criterion based on stress and strain with $\varepsilon_{\sigma_{1}}^{*}>\varepsilon_{\sigma_{2}}^{*}$ and $\sigma_{1}^{*}>\sigma_{2}^{*}$ : (a) 'stress criterion'; (b) 'strain criterion'; and (c) 'mixed criterion'.

- Modifying the treatment of the elements that change the state (from wrinkle or slack to taut). This is easily obtained considering them as being still in the 'old' state and applying a reduced penalization (Figure 5(c)).

$$
\text { new state is 'taut' } \rightarrow\left\{\begin{array}{l}
\text { old state is 'taut' } \rightarrow\left[\mathbf{D}_{\text {mod }}\right]=[\mathbf{D}] \\
\text { old state is not 'taut' } \rightarrow \begin{array}{l}
P=P_{\text {old }} \cdot \beta \\
\text { new state }=\text { old state }
\end{array}
\end{array}\right.
$$

This second modification (where $\beta=10$ usually provides good results) should be seen as a numerical artifice to avoid the oscillations. Even if it could be considered somehow nonphysical, it should be taken in account that this approximation disappears when the solution stabilizes as no further change of state for the elements is needed. 


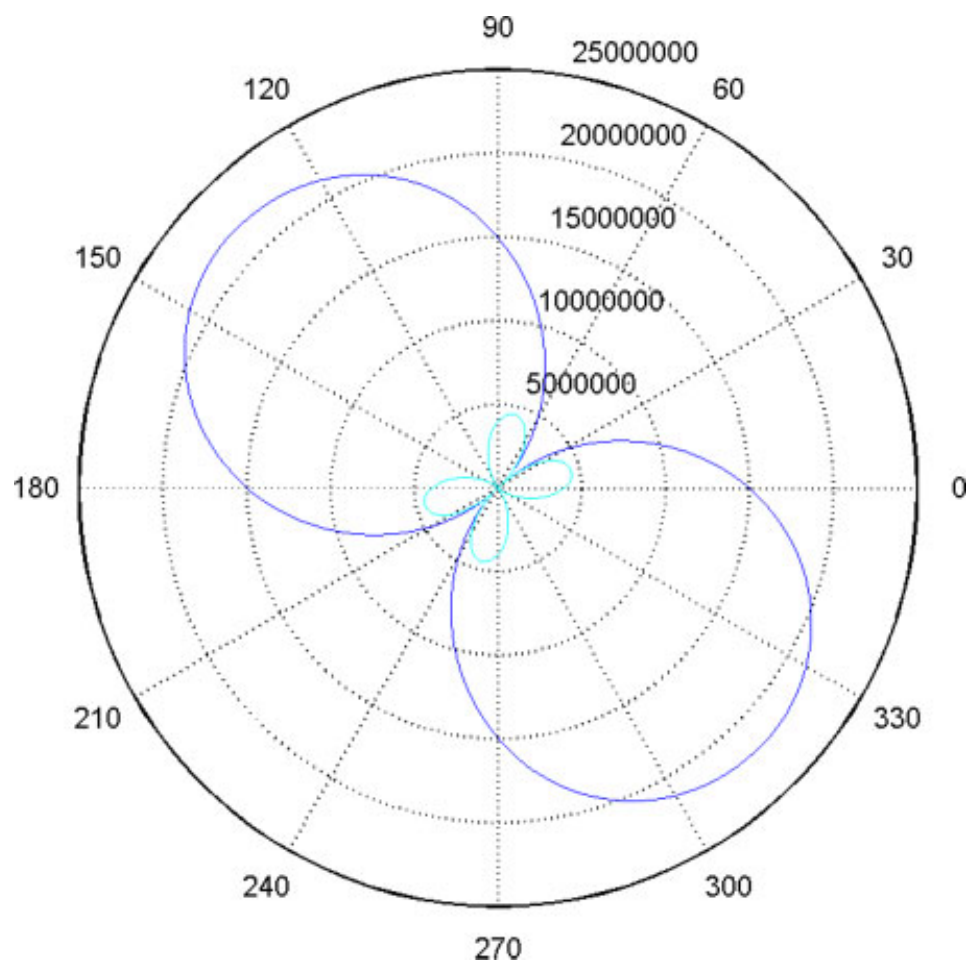

Figure 6. Isotropic penalize of material at $45^{\circ}$. Plot of principal stresses $\sigma_{1}$ and $\sigma_{2}$ for different orientations of the strain vector.

To gain insight in the effect of the material's correction it is interesting to consider a wrinkled material with a fixed wrinkling direction at $+45^{\circ}$. A uni-axial strain $\{\boldsymbol{\varepsilon}\}^{\mathrm{T}}=\left(\begin{array}{lll}\varepsilon_{11} & 0 & 0\end{array}\right)^{\mathrm{T}}$ it taken in account and the corresponding stress is calculated as $\{\boldsymbol{\sigma}\}=\left[\mathbf{C}_{\bmod }\right]\{\boldsymbol{\varepsilon}\}$ and used for the determination of the principal stresses $\sigma_{1}$ and $\sigma_{2}$. The same operation is performed after rotating the strain tensor of a small angle. Figure 6 presents a polar plot of the resulting principle stresses.

The plot shows how strain directions in a range of $\pm 10^{\circ}$ from the wrinkled direction end up with small compressive stresses while larger differences lead to sensibly wrong predictions.

The immediate consequence is that the correction of the material will remove correctly the compressive components only if after the softening the principal stresses did not change their orientation of more than around $10^{\circ}$. Unfortunately this is not the case during the early stages of the simulation when substantial redistribution can be expected, the aim of the proposed artificial stabilization is therefore to slow the change of stresses directions during the initial phases of the analysis.

\section{INCLUSION OF THE WRINKLING PROCEDURE IN THE SOLUTION PROCEDURE}

In the current work pseudo-dynamics procedure is systematically used to overcome the system's singularities. The key point is that the transient has no real interest and should be considered as 
a way to reach the final equilibrated configuration, which means that errors can be tolerated on the way to the final solution, as long as these errors disappear from the final result. Convergence should therefore considered as convergence of the whole solution process to the static solution and not as convergence of the single step to a compressive stresses free configuration.

In other words it is not considered essential to verify that no compressive stress appear during the solution process, but only that the final configuration has this characteristic.

Based on this observation, our proposed wrinkling correction scheme takes the form:

- solution of the pseudo-dynamic step;

- evaluation of stresses and strains;

- modification of the material;

- back to first point for the solution of the next time step.

The crucial aspects of the proposed procedure are

- Efficiency: the only additional cost is the correction of the material at the end of each solution step.

- Conservation of the elemental's convergence properties.

- Stabilization of the stress field provided by the dynamical process which together with the stabilization proposed for the material model effectively damps out the oscillations. The state of the membrane (taut, slack or wrinkled state) and consequently the elasticity tensor is considered constant during each time step minimizing the redistribution effect that follows to the softening of some directions. In this way the stiffness of the system is not subjected to 'brutal changes' during the Newton-Raphson iteration cicle allowing a much smoother convergence inside each solution step. Of course no guarantee is given that the single solution step will provide a 'compressive stress-free' solution, however, the oscillations are progressively damped out and the whole procedure tends toward a stable static solution. When the structural movement stops on the other hand, the strain field arrives to a constant value, no further change is therefore needed for the elasticity tensor which in turn guarantees that the compressive stresses are correctly removed.

A considerable amount of tests was carried out before obtaining a reliable procedure as the one described. Different approaches were tested and discarded as ineffective in particular:

- Any attempt to introduce effectively wrinkling as a non-linear material (varying during the Newton-Raphson iterations) met severe convergence problems. Even if an important effort was spent in the attempt of developing an effective stabilization, the introduction of the material non-linearity always spoiled the convergence properties, leading generally to a slow convergence; an increase in the number of iteration is reported even in Reference [11] in which the authors use a 'kinematic manipulation' and derive a consistent tangent stiffness matrix.

- The possibility of performing a subiteration loop to guarantee the correct removal of stresses (iteration of the solution and correction steps over the same time step) was considered and tested. This proved to be effective in many cases, showing however a very slow convergence in other situations. Since the computational effort was much greater and the final identical (static) solution, this possibility was discarded.

We remark in particular that the introduction of the dynamics is beneficial for the stability of any solution strategy as basically 'the solution cannot change much in a small time'. This 
source of stabilization is very 'cheap' as already needed for other reasons consequently the design of any wrinkling strategy should take the maximum possible advantage of it.

This beneficial effect is commonly recognized and it is at the base of the choice of pseudodynamic methods that are commonly introduced (see for example References $[6,7,9,10]$ ) to keep the advantages of the dynamics while allowing to converge directly to the static configuration.

\section{TEST EXAMPLES-PROCEDURE}

The aim of the chosen examples is to provide a validation of the wrinkling procedure. The idea is that the use of a sufficiently large number of elements should allow a proper description of the formation of the wrinkles, making the compressive stresses vanish. Basing on this, the results of the tests performed on large meshes are considered as reference solution and used as benchmarks in assessing the performance of the procedure.

In the following pages a number of test examples are described. All tests are carried out on different meshes with increasing mesh densities.

Buckling is initialized by the presence of imperfections. In the absence of out-of-plane loads it is necessary to provide an artificial disturbance to initialize the formation of the wrinkles. Different approaches are possible for the application of such disturbs, in which size and distribution are crucial particularly for shell based analysis. A possibility, as reported in Reference [10] is the application of a disturb corresponding to the first buckling mode and scaled depending on the thickness of the shell. Another possibility is to introduce a random out of plane disturb [9] on each node in the form, $z_{i}=\alpha \delta_{i} h$, where $\alpha$ is an a-dimensional amplitude parameter, $\delta_{i} \in[-1,1]$ is a pseudo-random number and $h$ is the membrane thickness. The same work [9] reports some guidelines for the choice of the amplitude parameter suggesting that values in the range $0.01<\alpha<1.0$ are large enough to start the formation of the wrinkles while leading to the same final wrinkling distribution. In purely membranal analysis as the one performed in this work no deterministic description of the wrinkling field is attempted consequently the only requirement is the introduction of an imperfection to start the out of plane deformation. In current work this was obtained by a couple of (very little) equilibrated forces applied on two adjacent nodes.

\subsection{Test 0 -rectangular membrane}

The aim of this test is to compare the result obtained with the enriched-element procedure with the other results reported in literature.

In particular, the test is performed using the scheme reported in Reference [28] for the example 1 and shown in Figure 7(a). A rectangular membrane of size $8 \times 4 \mathrm{~m}$ is fixed at its four sides; at the corners it is subjected to four tension loads $\left(P=0.001 \mathrm{~N}\right.$ with $\left.\varphi=45^{\circ}\right)$. The mesh is obtained by 64 elements and the wrinkling analysis direction is reported in Figure 7(b). The contour of the I and II PK2 stresses is shown in Figure 8(a) and (b). The maximum tensile stress occurs in the corners with a value of about $0.11 \mathrm{~Pa}$. Stress direction figures and values agree well with the result published in Reference [28]. At the end of the simulation elements state become wrinkled from first to fifth element, slack for the sixth and taut for seventh and eighth elements. 


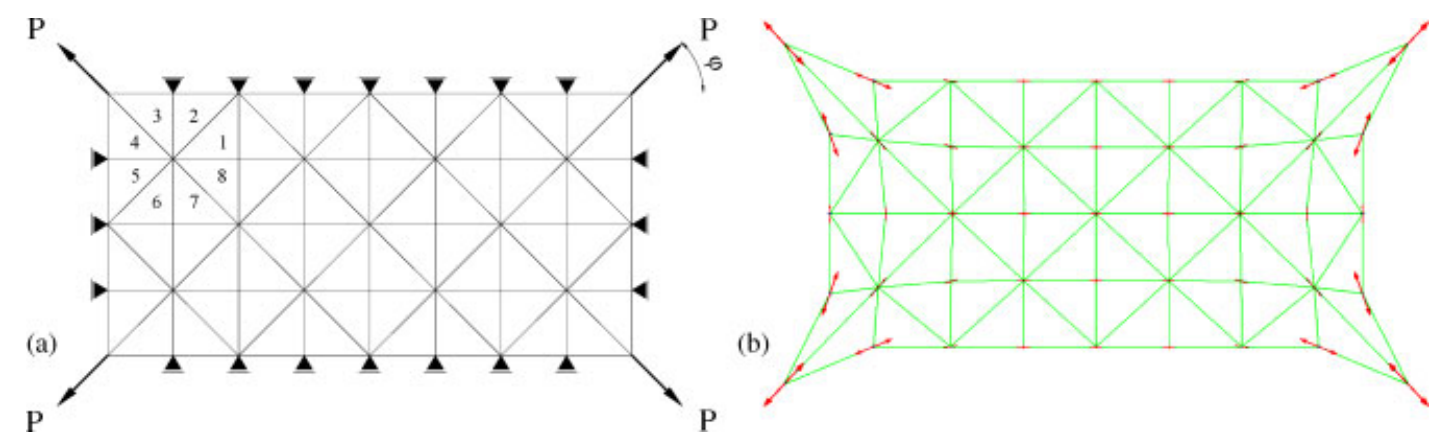

Figure 7. (a) Rectangular membrane scheme with four corner loads; and (b) principal stress direction PK2.

\subsection{Test 1-inflated circular airbag}

The inflation of a circular airbag is probably one of the best examples to be used in testing the efficacy of the wrinkling procedure. The simulation is carried out with the parameters reported in Figure 9. Symmetry conditions are used and the problem is evaluated with and without wrinkling algorithm. In particular the same airbag is simulated using three different meshes with a mesh density increasing progressively (coarse mesh with 236 normal elements Figure 10(a), and enriched element, Figure 10(c); medium mesh with 818 elements not shown and dense mesh with 4802 elements Figure 10(b)).

The use of a standard membrane element leads in this case to the growth of very high tangential compressive stresses. In this situation a very dense mesh is needed to capture the formation of folds and wrinkles that eliminate the compression, Figure 10(a) and (b). The comparison of Figure 10(a) and (b) suggests immediately how some deep folds (larger wrinkles) appear in the solution. In the figures, PK2 stresses are plotted showing both stresses magnitude and orientation.

The analysed problem is axi-symmetric and only a quadrant of the air-bag is simulated, but the result in Figure 10(a) and (b) is not. As described above, the presence of very high tangential compressive stresses (meridian stress), when classical membranes are used, lead to the development of local buckling and to growth of wrinkles; the compressive stress becomes zero and only stress on the parallels remains. This effect (reset of the compressive stress) depends on the localization of the first buckling zone (function of the element dimension, mesh discretization, very small error introduced into the code, etc.) and for this reason the position cannot be defined a priori and the process is very close to be random. It can be easily checked how even different runs of the same structure with the same mesh can lead to different wrinkling patterns. Folds can be either located in some sections only (similar result is obtained in Reference [11] for the air-bag) if this is enough to eliminate the meridian compressive stress, Figure 10(b), or spread on the surface, Figure 10(a). It is necessary to remember that the aim of the simulation is to obtain a solution without compressive stress and not to describe the wrinkles formation. The comparison is between two solutions where the reference terms are stress, displacement, etc. but not the dimension of wrinkles or folds.

It deserves attention to notice how in the two cases folds location changes but their distance tends to be the same. In Figure 10(b) for dense mesh three big folds are developed and distance between the I and II fold and between the II and III fold is very similar. 


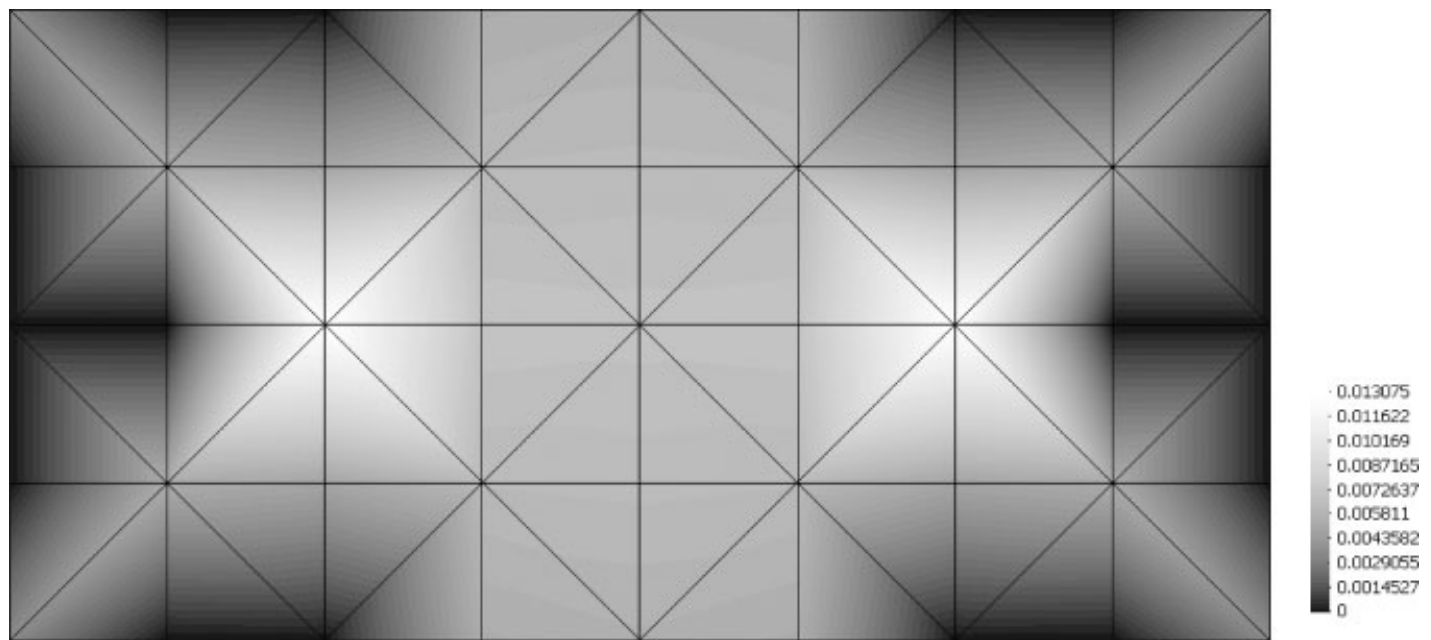

(a)

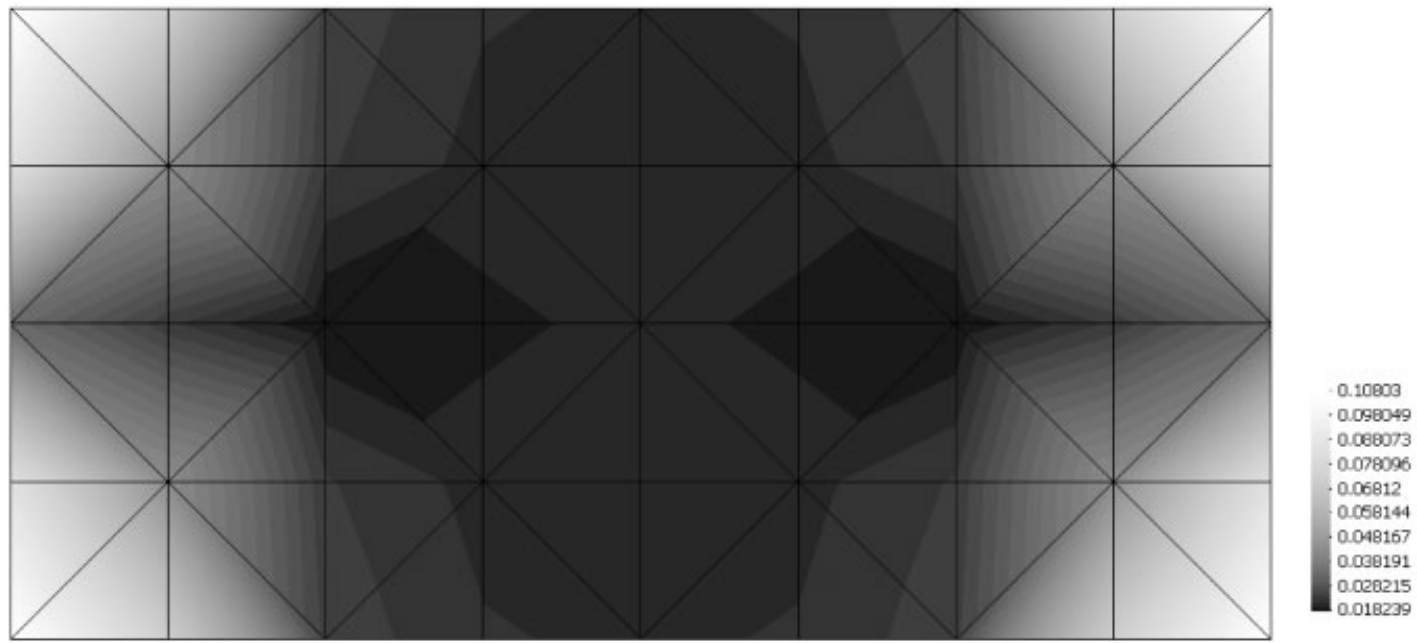

(b)

Figure 8. (a) Contour of the PK2 stress (Pa) Ist stress; and (b) IInd stress.

The introduction of wrinkling algorithm clearly shows how the essence of the behaviour is correctly described even using much coarser meshes in terms of the maximum displacement of the central point and of the principal tension stress, Figure 10(c). It is worth noting how, in Table II, only the densest mesh can catch the behaviour obtained by wrinkling algorithm, leading to the same value of stresses and displacement at the centre of the airbag. A coarse mesh with enriched or wrinkled element leads to a very good result both in terms of stresses and of displacements while only the densest mesh appears to describe the phenomena in the correct way. 

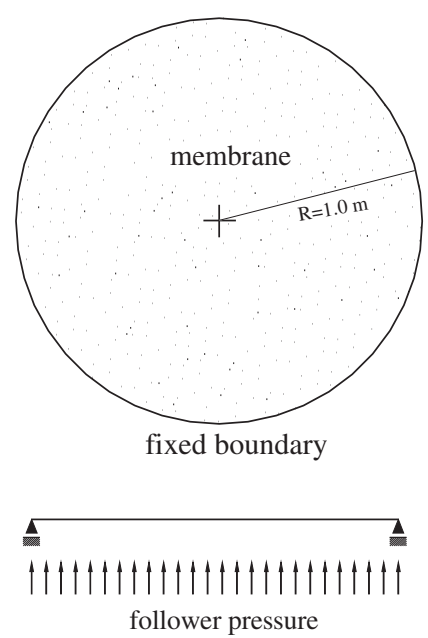

inflated circular airbag parameter:

Size $\mathrm{R}=1000 \mathrm{~mm}$

Size $B=1000 \mathrm{~mm}$

thickness of membrane $=0.001 \mathrm{~mm}$;

material constants:

Young module $\mathrm{E}=7000 \mathrm{~Pa}$;

Poisson coefficient $v=0.3$

Density $\rho=2700 \mathrm{Kg} / \mathrm{m}^{3}$

The boundary nodes are free in the plane and are fix only the displacement $\mathrm{Z}$.

Figure 9. Scheme of inflated circular airbag membrane.
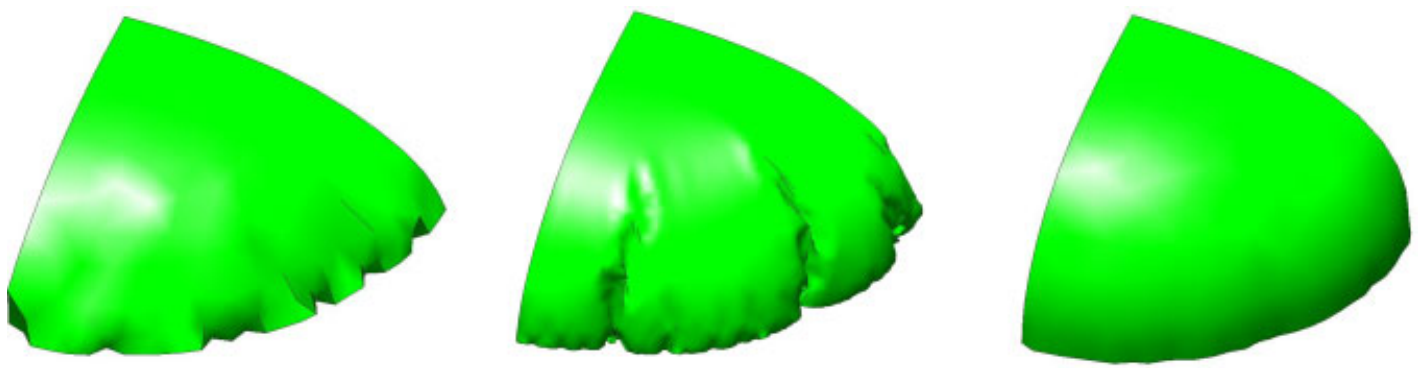

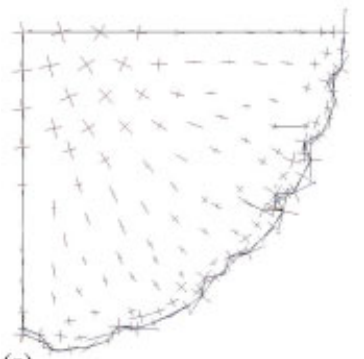

(a)

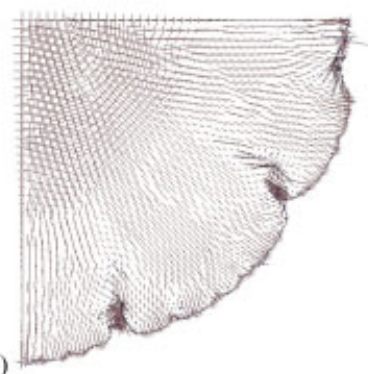

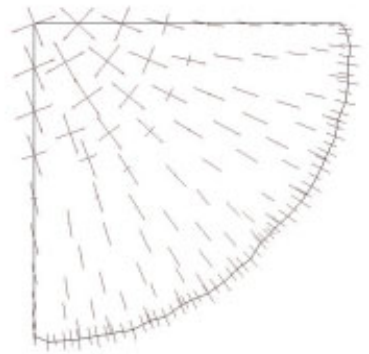

(c)

Figure 10. Three dimension views of inflated airbag and principal stresses: (a) coarse (236 el.); (b) dense mesh (4802 el.) with normal element; and (c) coarse mesh (236 el.) with enriched element.

\subsection{Test 2-shear test}

The shear test is applied by imposing the displacement of the side of a square membrane. The simulation is performed using the scheme and parameters reported in Figure 11. As boundary conditions, the lower line boundary is fixed and the superior line boundary has imposed displacement $(u=200 \mathrm{~mm})$. 
Two cases are reported: one with wrinkling algorithm (coarse mesh, Figure 12) and one without (dense mesh, Figure 13). The final result, that is the formation of only one diagonal tension zone, is correctly met in both simulations. As expected in the first simulation, Figure 12(a)

Table II. Second Piola-Kirchhoff (PK2) stresses and vertical displacement at the centre of the membrane.

\begin{tabular}{lcccccc}
\hline & PK2 & Dense (Pa) & Coarse (Pa) & & Dense $(\mathrm{m})$ & Coarse (m) \\
\hline Normal element & $S_{\mathrm{I}}$ & $9.55 \mathrm{e} 7$ & $11.3 \mathrm{e} 7$ & Vert. & 0.46531 & 0.37282 \\
& $S_{\text {II }}$ & $9.05 \mathrm{e} 7$ & $10.5 \mathrm{e} 7$ & Disp. & & \\
Enriched element & $S_{\mathrm{I}}$ & $9.58 \mathrm{e} 7$ & $9.51 \mathrm{e} 7$ & Vert. & 0.47075 & 0.47009 \\
& $S_{\text {II }}$ & $9.56 \mathrm{e} 7$ & $9.09 \mathrm{e} 7$ & Disp. & & \\
\hline
\end{tabular}

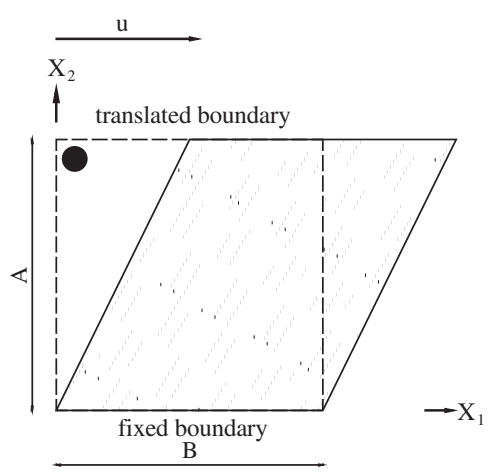

$$
\begin{aligned}
& \text { shear test parameter: } \\
& \text { Size } \mathrm{A}=1000 \mathrm{~mm} \\
& \text { Size } \mathrm{B}=1000 \mathrm{~mm} \\
& \text { thickness of membrane }=0.001 \mathrm{~mm} \text {; } \\
& \text { material constants: } \\
& \text { Young module } \mathrm{E}=7000 \mathrm{~Pa} \text {; } \\
& \text { Poisson coefficient } \mathrm{v}=0.3 \\
& \text { Density } \rho=2700 \mathrm{Kg} / \mathrm{m}^{3}
\end{aligned}
$$

Figure 11. Scheme of shear test membrane.

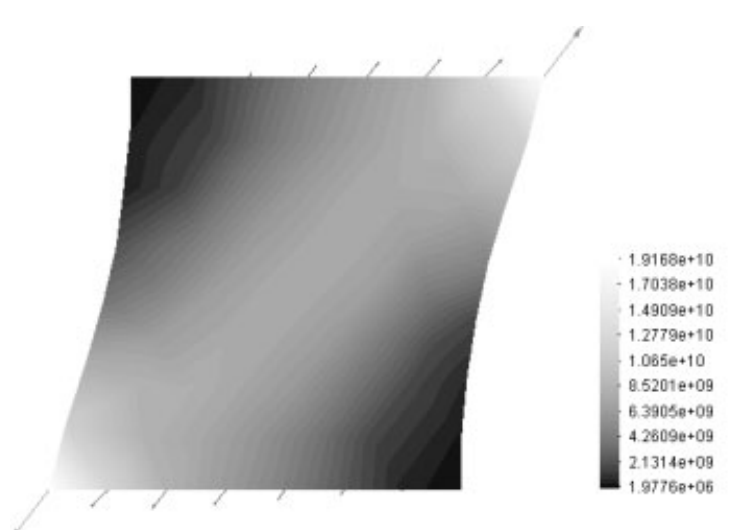

(a)

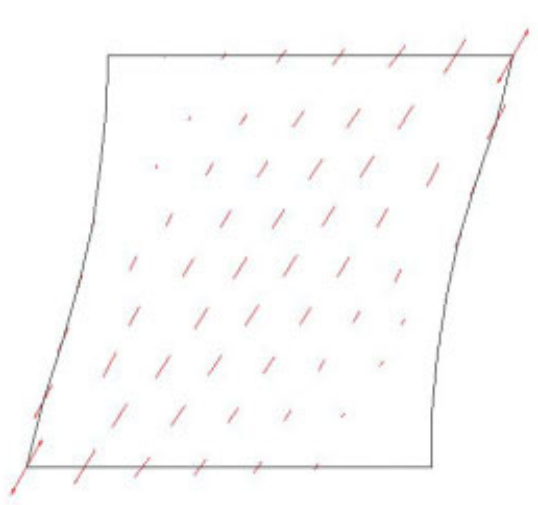

(b)

Figure 12. Coarse mesh (98 elem.) with enriched element: (a) contour of the PK2 stress (MPa); and (b) principal direction. 


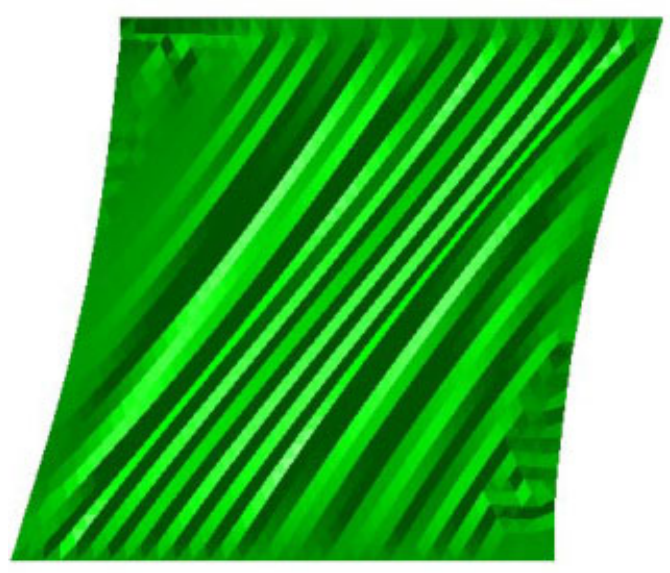

(a)

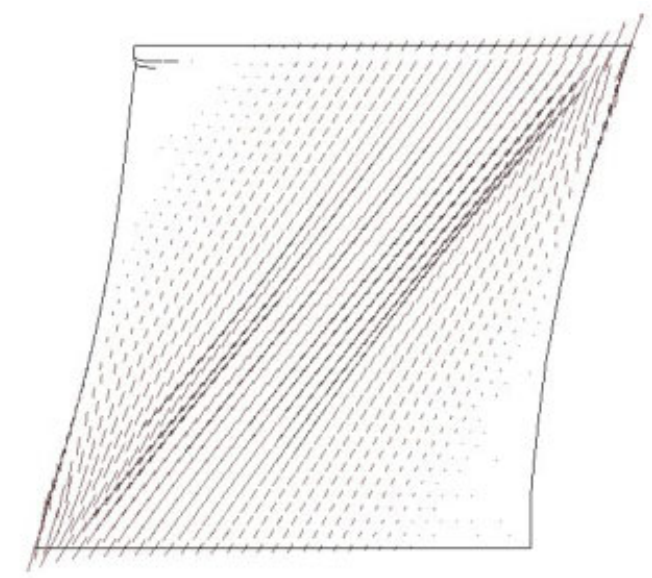

(b)

Figure 13. Dense mesh (2450 elem.) with normal element: (a) displacement out of the plane; and (b) principal direction.
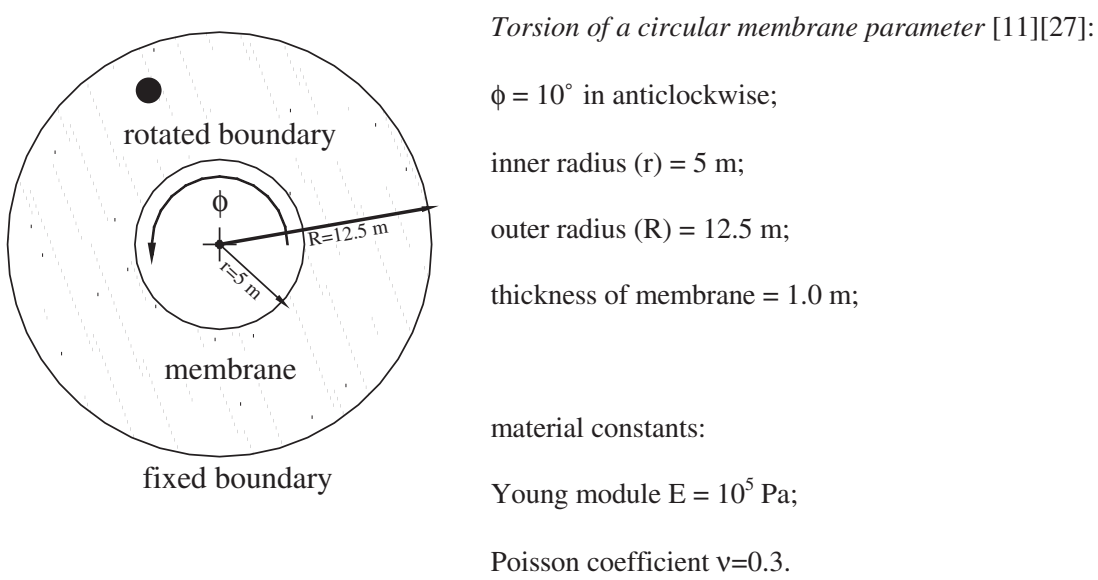

Figure 14. Scheme of torsion circular annulus-shaped membrane.

and (b) (coarse mesh), the enriched element changes its own state from taut to wrinkled while the second simulation, Figure 13(a) and (b) (dense mesh), shows the development of a pattern of 'waves' (wrinkles). As proposed in the introduction a small disturb is applied to the top left corner of the structures to initialize the simulation. The contours of the displacement, Figure 13(a) and (b), show a minor difference between the top-left corner and the bottom-right corner which can be attributed to the local perturbation introduced into the simulation and to the smoothing process of the displacement.

The same difference in the results obtained by combined membrane simulation with outof-plane and initial imperfections is typical and reproduced by many other authors (e.g. Reference [9]). 


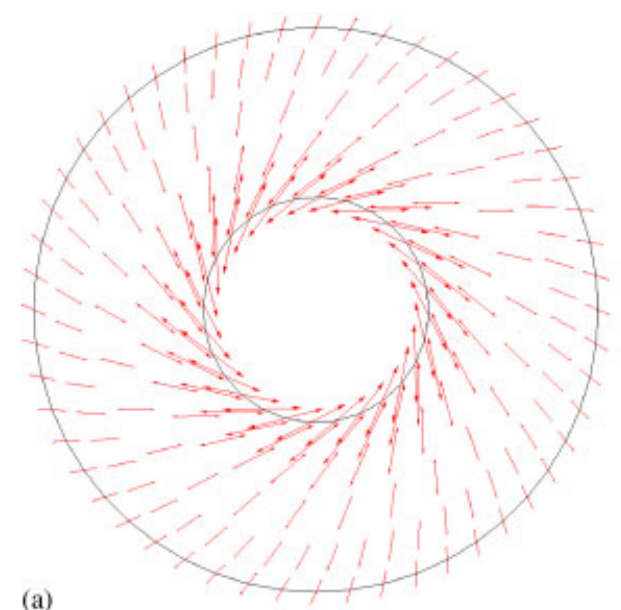

(b)

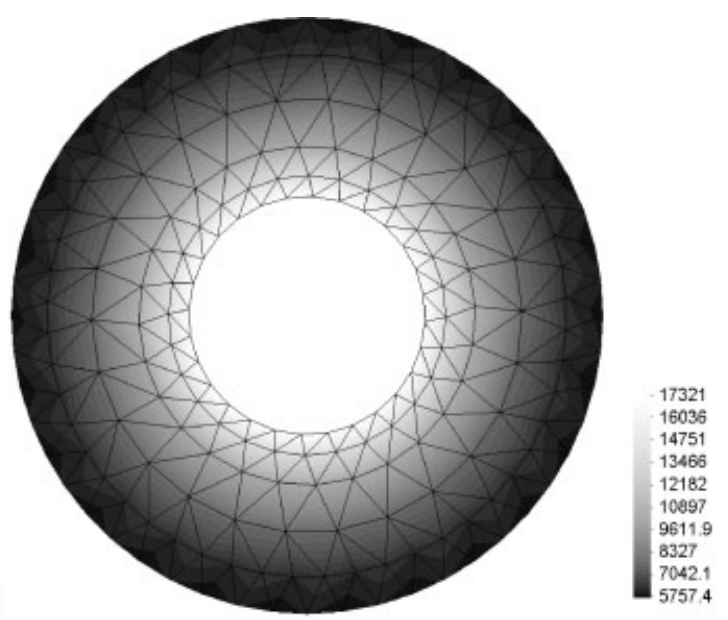

Figure 15. Circular annulus-shaped membrane with coarse mesh (348 elem.) and enriched element: (a) principal stress direction; and (b) contour of the PK2 stress (MPa).

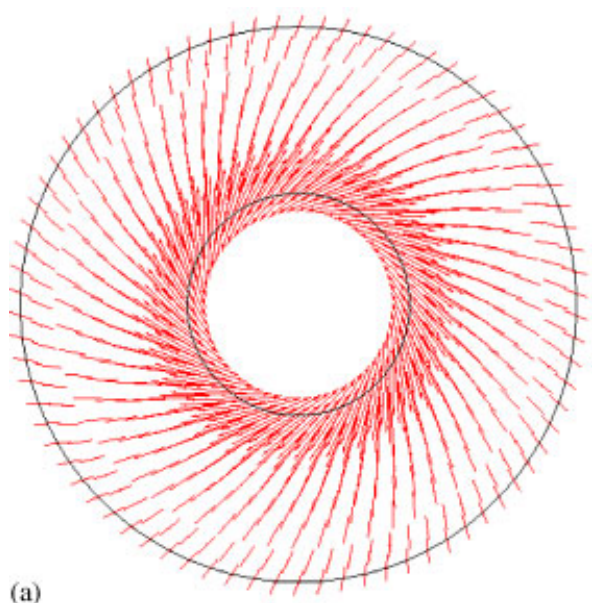

(b)

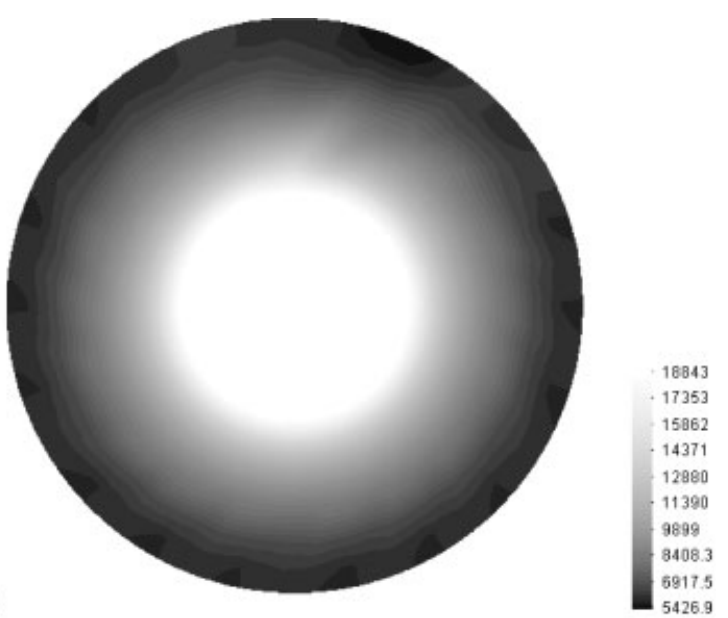

Figure 16. Circular annulus-shaped membrane with dense mesh (1254 elem.) and enriched element: (a) principal stress direction; and (b) contour of the PK2 stress (MPa).

\subsection{Test 3-torsion of a circular membrane}

A circular annulus-shaped membrane is attached to a rigid disk (for the behaviour contained into the membrane plane) at the inner edge and to a guard ring at the outer edge (e.g. References [11,27]). The turning of the rigid disk causes the wrinkling of the membrane, Figure 14. In the following pictures the solutions of three simulations are reported: coarse (Figure 15), dense mesh with the enriched elements (Figure 16) and a very dense mesh with normal elements (Figure 17). 


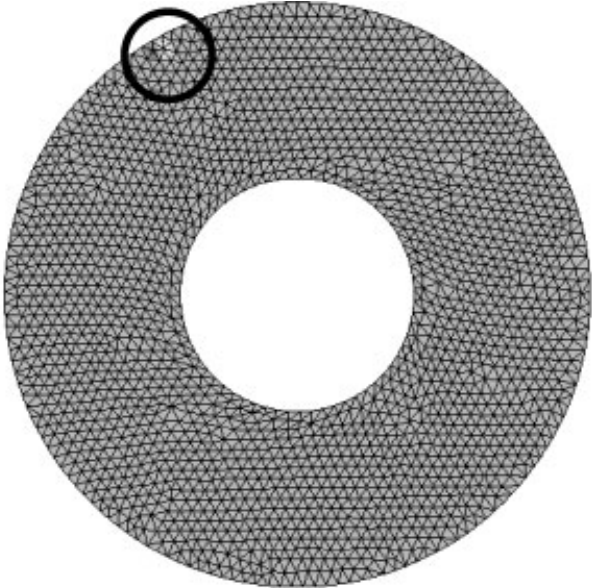

(a)

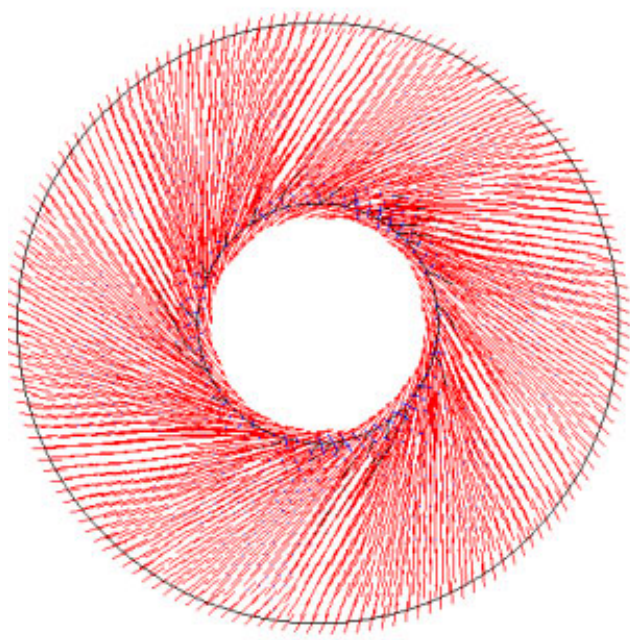

(c)

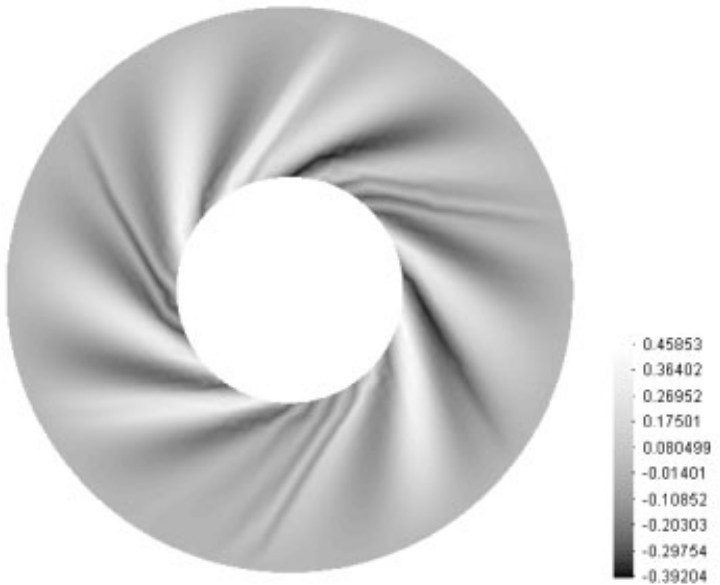

(b)

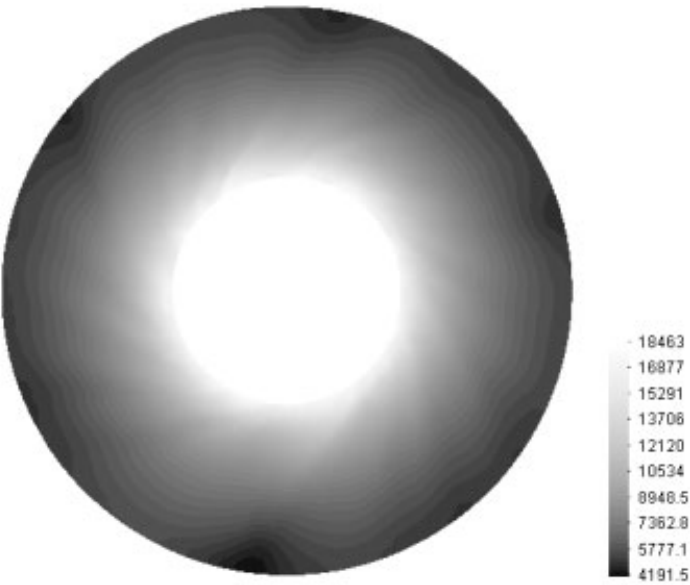

(d)

Figure 17. Circular annulus-shaped membrane with very dense mesh (3736 elem.) and normal element: (a) finite element mesh with initial perturbation; (b) deformed shape out of the plane; (c) principal stress direction; and (d) contour of the PK2 stress (MPa).

Principal stress direction and contour of the PK2 stress (MPa) are shown in Figures 15(a) and (b) and 16(a) and (b). The vectors in the node represent the magnitude of the principal stresses. As expected, only one vector appears on each node that represents the non-zero tensile principal stress.

The maximum tensile stress is on the inner edge with a value of $17321 \mathrm{MPa}$, Figure $15(\mathrm{~b})$, for coarse and $18843 \mathrm{MPa}$, Figure 16(b). These values agree with previously published results [27]. The step from coarse to dense mesh does not change the principal aspect of the simulation (the stress is only positive, the qualitative distribution of the direction is the same, all elements 
change their own state: initial state is taut, last state is wrinkled), but only some adjustments on the PK2 stress value are performed.

A small disturb is applied to the top-left corner of the structures to initialize the simulation with very dense mesh, Figure 17(a). During the transient of the dynamic relaxation there is the development of the pattern of 'waves' and the last configuration is shown in Figure 17(b). The wrinkles are distributed all over the circular annulus-shaped membrane and a similar configuration is obtained when the position of the disturb is changed. The direction of the principal stress, Figure 17(c), is very similar to Figures 15(a) and 16(a), as well as the contour of the PK2 stress. The maximum stress is about $18500 \mathrm{MPa}$, Figure $17(\mathrm{~d})$, close to the value carried out by wrinkling simulation with dense mesh. The stress distribution remains constant on $\theta$ and the difference represented by the contour in Figures 15-17 (more evident near the outer edge into the last element of the mesh) is due only to the smoothing of the stress into the element.

\section{CONCLUSIONS}

The present paper describes the application of the finite element method to the analysis of membrane with wrinkling. In the first part, a finite element membrane with non-linear geometric behaviour is reviewed. The second part describes the modification procedure and stabilization for the material, together with its inclusion in a stabilized pseudo-dynamic solution procedure. Finally, the third part is an overview of same examples performed, with the discussion of the numerical aspect of the analysis. Some considerations about the introduction of imperfection, the problem of the convergence of the iteration process and comparison with other results are then developed.

In authors' opinion the main stabilization is given by the dynamic relaxation introduced in the process. In the development of new strategies it is therefore advisable to take full advantage of this characteristic.

It is important to remark here how the proposed method is designed for the simulation in conditions of large displacements and small strains in which the PK2 stresses are basically a rotation of the Cauchy ones. The results are however very interesting even when, as for the annulus, the deformations are not small. This suggests that the procedure could be effectively extended to more general material models.

\section{REFERENCES}

1. Rossi R, Lazzari M, Vitaliani R, Oñate E. Convergence of the modified material model for wrinkling simulation of light-weight membrane structures. In Textile Composites and Inflatable Structures (Structural Membranes 2003), Oñate E, Kröplin B (eds). CIMNE: Barcelona, 2003.

2. Lazzari M, Saetta AV, Vitaliani RV. Non-linear dynamic analysis of cable suspended structures subjected to wind actions. Journal of Computer and Structures 2001; 79:954-968.

3. Lazzari M. Geometrically non-linear structures subjected to wind actions. Ph.D. Dissertation, University of Padua, Italy, 2002.

4. Cirak F, Ortiz M, Schroeder P. Subdivision surfaces: a new paradigm for thin shell finite element analysis. International Journal for Numerical Methods in Engineering 2000; 47:2039-2072.

5. Cirak F, Ortiz M. Fully C1 conforming subdivision elements for finite deformation thin shell analysis. International Journal for Numerical Methods in Engineering 2001; 51:813-833.

6. Flores F, Onate E. Rotation free element for finite strain analysis of elastic-plastic thin shell and membrane structures. Textile Composites and Inflatable Structures. CIMNE: Barcelona, 2003. 
7. Wong YW, Pellegrino S. Computation of wrinkling amplitudes in thin membranes. 43rd AIAA/ASME/ASCE/AHS/ASC Conference, Denver, 2002.

8. Wong YW, Pellegrino S, Park KC. Prediction of wrinkle amplitudes in square solae sails. 44th AIAA/ASME/ASCE/AHS/ASC Conference, Norfolk, 2003.

9. Tessler A, Sleight DW, Wang T. Nonlinear shell modeling of thin membranes with emphasis on structural wrinkling. 44th AIAA/ASME/ASCE/AHS/ASC Conference, Norfolk, 2003.

10. Su X, Abdi F, Taleghani B, Blandino J. Wrinkling analysis of a kapton square membrane under tensile loading. 44th AIAA/ASME/ASCE/AHS/ASC Conference, Norfolk, 2003.

11. Accorsi M, Lu K, Leonard J. Finite element analysis of membrane wrinkling. International Journal for Numerical Methods in Engineering 2001; 50:1017-1038.

12. Liu X, Jenkins C, Schur W. Large deflection analysis of pneumatic envelopes using a penalty parameter modified material model. Finite Elements in Analysis and Design 2001; 37:233-251.

13. Bathe KJ. Finite Element Procedures. Prentice-Hall: Englewood Cliffs, NJ, 1996.

14. Belytschko T. Nonlinear Finite Elements for Continua and Structures. Wiley: Chichester, 2000.

15. Zienkiewicz OC, Taylor RL. The Finite Element Method, 1: The Basi, 2: Solid Mechanics, 3: Fluid Dynamics (5th edn). Butterworth-Heinemann: Stoneham, MA, 2000.

16. Stanuszek M. FE analysis of large deformations of membranes with wrinkling. Finite Elements in Analysis and Design 2003; 39(7):599-618.

17. Taylor RL. Finite element analysis of membrane structures. CIMNE Internal Report, 2001.

18. Valdes G. Analisys de membranas. CIMNE Internal Report, 2002.

19. Rossi R. Analysis of membranes with wrinkles. CIMNE Internal Report, 2003.

20. Brannon RM. Elementary vector and tensor analysis. University of New Mexico Report, Albuquerque, 2002.

21. Barnes MB. Form-finding and analysis of prestressed nets and membranes. Journal of Computer and Structures 1988; 30(3):685-695.

22. Ong CF, Wakfeld DS, Barnes MR. Interactive graphic CAD for tension structure. Journal of Computer and Structures 1991; 41(6):1305-1312.

23. Fujikake M, Kojima O, Fukushima S. Analysis of fabric tension structures. Journal of Computer and Structures, Pergamon, 1989; 32:537-547.

24. Tabarrok B, Qin Z. Nonlinear analysis of tension structures. Journal of Computer and Structures 1992; 45(5/6):973-984.

25. Contri P, Schrefler B. A geometrically non-linear finite element analysis of wrinkled membrane surfaces by a no compression material model. Communications in Applied Numerical Methods 1988; 4:5-15.

26. Roddeman DG, Oomens CWJ, Janssen JD, Drukker J. The wrinkling of thin membranes. Part 1: theory. Journal of Applied Mechanics 1987; 54:884-887.

27. Roddeman DG, Oomens CWJ, Janssen JD, Drukker J. The wrinkling of thin membranes. Part 2: numerical analysis. Journal of Applied Mechanics 1987; 54:888-892.

28. Ding H, Yang B. The modelling and numerical analysis of wrinkled membranes. International Journal for Numerical Methods in Engineering 2003; 58:1785-1801.

29. Roddeman DG. The wrinkling of thin membranes. Part 2: numerical analysis. Communication in Applied Numerical Methods 1991; 7:299-307. 